Pure and Applied Mathematics Quarterly

Volume 2, Number 4

(Special Issue: In honor of

Robert MacPherson, Part 2 of 3)

1103-1130, 2006

\title{
Balanced Normal Cones and Fulton-MacPherson's Intersection Theory
}

\author{
Allen Knutson \\ Dedicated to Bob MacPherson on the occasion of his 60th birthday
}

\begin{abstract}
Let $X$ be a subscheme of a reduced scheme $Y$. Then $Y$ has a flat degeneration to the normal cone $\mathrm{C}_{\mathrm{X}} \mathrm{Y}$ of $\mathrm{X}$, and this degeneration plays a key step in Fulton and MacPherson's "basic construction" in intersection theory. The intersection product has a canonical refinement as a sum over the components of $C_{X} Y$, for $X$ and $Y$ depending on the given intersection problem. The cone $C_{X} Y$ is usually not reduced, which leads to the appearance of multiplicities in intersection formulae.

We describe a variant of this degeneration, due essentially to Samuel, Rees, and Nagata, in which $Y$ flatly degenerates to the "balanced" normal cone $\overline{\mathrm{C}}_{X} \mathrm{Y}$. This space is reduced, and has a natural map onto the reduction $\left(C_{X} Y\right)_{\text {red }}$ of $C_{X} Y$. The multiplicity of a component now appears as the degree of this map. Hence intersection theory can be studied using only reduced schemes. Moreover, since the map $\bar{C}_{X} Y \rightarrow\left(C_{X} Y\right)_{\text {red }}$ may wrap several components of $\overline{\mathrm{C}}_{X} \mathrm{Y}$ around one component of $\mathrm{C}_{X} \mathrm{Y}$, writing the intersection product as a sum over the components of $\overline{\mathrm{C}}_{X} \mathrm{Y}$ gives a further canonical refinement.

In the case that $X$ is a Cartier divisor in a projective scheme $Y$, we describe the balanced normal cone in homotopy-theoretic terms, and prove a useful upper bound on the Hilbert function of $\overline{\mathrm{C}}_{\mathrm{X}} \mathrm{Y}$.
\end{abstract}

\section{Contents}

1. Introduction

1.1. Normal cones and balanced normal cones

1.2. The maps gr $\mathrm{R} \rightarrow \overline{\mathrm{gr}} \mathrm{R}$ and $\overline{\mathrm{C}}_{\mathrm{W}} \mathrm{V} \rightarrow \mathrm{C}_{\mathrm{W}} \mathrm{V}$ 
1.3. The "basic construction" in intersection theory

1.4. Acknowledgements

2. Properties of $\overline{\mathrm{gr}}$

2.1. Rees' formula for $\bar{q}$

3. Some normal cones and balanced normal cones as flat limits

4. The Cartier case: the ring $\widetilde{g r}$ and a homotopy interpretation

\section{INTRODUCTION}

1.1. Normal cones and balanced normal cones. Let $R$ be a commutative Noetherian ring with unit - indeed, all rings encountered this paper will have these properties - and let I be an ideal. For $r \in R$, define $q(r)$ as the largest $n$ such that $r \in I^{n}$, or $\infty$ if there is no largest $n$ (e.g. if $\left.r=0\right)$. Then the associated graded ring is defined as

$$
\operatorname{gr} R:=\bigoplus_{n \in \mathbb{N}}\{r: q(r) \geq n\} /\{r: q(r) \geq n+1\} .
$$

One of its virtues is that it has a map not only to, but from, R/I. (Whereas $R$ doesn't naturally have a map from R/I.) Moreover, the map $R / I \rightarrow g r R$ is an inclusion (as the $\mathrm{n}=0$ summand).

Following Samuel (our reference is Rees' book [Re]), define $\overline{\mathbf{q}}(\mathrm{r}):=\lim _{\mathfrak{n} \rightarrow \infty} \mathrm{q}\left(\mathrm{r}^{\mathfrak{n}}\right)$ $/ \mathrm{n}$, the homogenization of the filtration $\mathrm{q}$. Samuel proved that this limit exists. Nagata and Rees showed that it is rational-valued with bounded denominator. Rees gave a formula for $\overline{\mathrm{q}}$, using Rees algebras, which we recall in section 2 in the cases we need. Nagata proved that $\bar{q}-\mathrm{q}$ is bounded [Re, theorem 4.21], which implies that

$$
\overline{\mathrm{gr}} \mathrm{R}:=\bigoplus_{\mathrm{n} \in \mathbb{Q}}\{\mathrm{r}: \overline{\mathrm{q}}(\mathrm{r}) \geq \mathrm{n}\} /\{\mathrm{r}: \overline{\mathrm{q}}(\mathrm{r})>\mathrm{n}\}
$$

is again Noetherian $[R e$, lemma 2.46]. Note that this grading is by $\mathbb{Q}$, not (usually) by $\mathbb{N}$. 
Example 1. Let $\mathrm{R}=\mathbb{F}[\mathrm{x}], \mathrm{I}=\left\langle\mathrm{x}^{2}\right\rangle$. (Throughout the paper $\mathbb{F}$ denotes an arbitrary field.) Then $\mathrm{q}\left(\mathrm{x}^{\mathrm{n}}\right)=\lceil\mathrm{n} / 2\rceil$, and $\operatorname{gr} \mathrm{R} \cong \mathbb{F}\left[\bar{x}_{(0)}, \mathrm{y}_{(1)}\right] /\left\langle\bar{x}^{2}\right\rangle$, where the subscripts indicate the degrees. Whereas $\bar{q}\left(x^{n}\right)=n / 2$, and $\overline{\operatorname{gr}} \mathrm{R}=\mathbb{F}\left[\overline{\mathrm{x}}_{(1 / 2)}\right]$, or $\mathbb{F}\left[\bar{x}_{(1 / 2)}, \mathrm{y}_{(1)}\right] /\left\langle\bar{x}^{2}-\mathrm{y}\right\rangle$ written for comparison.

We urge the reader to check the details of this example, as examples 2 and 4 build upon this one.

The following intuition seems to be useful. In gr R in the example above, $x$ is "rushed" into the degree 0 piece (rather than waiting until degree $1 / 2$ where it "belongs"), and by degree 0 standards its square (which has $q=1$ ) vanishes. It is this premature appearance of $x$ that leads to its nilpotency in gr $R$.

\section{Proposition 1. (1) There is a flat degeneration of $\mathrm{R}$ to gr $\mathrm{R}$.}

(2) $\overline{g r} \mathrm{R}$ has no nilpotents.

(3) There is a flat degeneration of $\mathrm{R}$ to $\overline{\mathrm{gr}} \mathrm{R}$.

(4) There are natural maps gr $\mathrm{R} \leftrightarrow \operatorname{gr} \mathrm{R}_{0}=\mathrm{R} / \mathrm{I}$.

(5) There are natural maps $\overline{\mathrm{gr}} \mathrm{R} \leftrightarrow \overline{\mathrm{gr}} \mathrm{R}_{\mathrm{O}}=\mathrm{R} / \sqrt{\mathrm{I}}$.

(6) If $\mathrm{R}^{\bullet}$ is the homogeneous coordinate ring of a projective scheme over a field $\mathbb{F}=\mathrm{R}^{0}$, and $\mathrm{I}$ is graded, then the degeneration of $\mathrm{R}$ to $\operatorname{gr} \mathrm{R}$ is locally free. If $\mathrm{R}$ is also reduced, then the degeneration of $\mathrm{R}$ to $\overline{\mathrm{gr}} \mathrm{R}$ is also locally free.

Recall that a family is locally free if its coordinate ring is not only torsion free over the base (in this case $\mathbb{F}[t]$ ), but actually free.

Proof. We start with the second claim. Let $0 \neq \bar{r} \in \overline{g r} R_{n}$ be nilpotent, so $\bar{r}^{M}=0$. Then $\overline{\mathrm{q}}\left(\mathrm{r}^{\mathrm{M}}\right)>M n$. Hence $\overline{\mathrm{q}}(\mathrm{r})>\mathrm{n}$, a contradiction.

Then the first and third claims use the Rees algebras (which will reappear in section 6)

$$
\bigoplus_{n \in \mathbb{Z}} t^{-n}\{r: q(r) \geq n\} \quad \bigoplus_{n \in \mathbb{Z}} t^{-n}\{r: \bar{q}(r) \geq n / N\}
$$

inside $R\left[t, t^{-1}\right]$ to provide the flat families [Ei, sec. 6.5]. Here $N$ is the least common (or any other) multiple of the denominators occurring in $\overline{\mathrm{q}}$.

The fourth claim is obvious. For the fifth, we need to compute $\overline{\mathrm{gr}} \mathrm{R}_{0}=\mathrm{R} /\{\mathrm{r}$ : $\overline{\mathrm{q}}(\mathrm{r})>0\}$. Then

$$
\{\mathrm{r}: \overline{\mathrm{q}}(\mathrm{r})>0\}=\left\{\mathrm{r}: \exists M, \mathrm{q}\left(\mathrm{r}^{\mathrm{M}}\right)>0\right\}=\sqrt{\mathrm{I}} .
$$

If $R^{\bullet}$ is the coordinate ring of a projective variety over $\mathbb{F}$, then (1) each of its graded pieces are finite-dimensional and $(2) \cap_{j}^{\infty} I^{j}=\{0\}$. With these, we can lift an $\mathbb{F}$-basis of gr $\mathrm{R}$ to an $\mathbb{F}[\mathrm{t}]$-basis of the coordinate ring of the first family. For the second family, we need $\sqrt{\cap_{j}^{\infty} I^{j}}=\{0\}$, namely that $R$ is reduced. 
Much of this paper is concerned with the natural map gr $R \rightarrow \overline{g r} R$, which we take up in the next section.

Young algebraic geometers are strictly indoctrinated to regard killing nilpotents as a bad habit; information is being thrown away. To allay their suspicions, we emphasize that $\overline{\text { gr }} \mathrm{R}$ is not just gr $\mathrm{R}$ mod its nilpotents (though as we shall see, it contains that as a subring). If $R$ and I are graded so that one can speak of Hilbert functions, then $R, g r R, \overline{g r} R$ all have the same Hilbert function, whereas gr $\mathrm{R}$ modulo its nilpotents will have a smaller Hilbert function (unless it had no nilpotents). In this sense, the information usually recorded in nilpotents is just showing up in a different way.

Since the denominators in $\overline{\mathrm{q}}$ are bounded, one may be tempted to clear them by rescaling the grading. This seems to carry no benefit, and only serves to make the map gr $\mathrm{R} \rightarrow \overline{\text { gr }} \mathrm{R}$ no longer graded.

If $V=$ Spec $R$ and $W=$ Spec $R / I$, then the space Spec gr $R$ is called the normal cone $C_{W} V$. We christen the space Spec $\overline{g r} R$ the balanced normal cone $\bar{C}_{W} V$, where the term "balanced" is chosen to evoke the idea that the grading is carefully weighted to avoid creating nilpotents.

Much as in the case of ordinary normal cones, it is not hard to define balanced normal cones for arbitrary pairs of schemes $\mathrm{W} \subseteq \mathrm{V}$ where $\mathrm{V}$ is reduced. As all our examples are Specs or Projs, we defer the details of globalization to section 5. As a bonus, we define in that section the "balanced blowup" along $W$ in the case $\mathrm{V}$ is of finite type.

In a future paper $[\mathrm{AK}]$, we will generalize the construction from this paper to a construction that completes essentially any one-parameter family of reduced schemes, the case studied in this paper being the family giving the degeneration to the normal cone.

1.2. The maps gr $R \rightarrow \overline{\text { gr }} R$ and $\bar{C}_{W} V \rightarrow C_{W} V$. Since $\overline{g r} R$ has no nilpotents, the kernel of $\beta: \operatorname{gr} R \rightarrow \overline{g r} R$ is plainly at least the nilpotents.

Proposition 2. The kernel of $\beta: \operatorname{gr} \mathrm{R} \rightarrow \overline{\mathrm{gr}} \mathrm{R}$ is exactly the nilpotent elements in gr R. If gr $\mathrm{R}$ has no nilpotents, then $\beta$ is an isomorphism (and otherwise not).

Proof. We need the calculation

$$
\overline{\mathrm{q}}(\mathrm{r})>\mathrm{n} \Longleftrightarrow \exists \mathrm{m}, \overline{\mathrm{q}}(\mathrm{r})>\mathrm{n}+\frac{1}{\mathrm{~m}} \Longleftrightarrow \exists M>0, \mathrm{q}\left(\mathrm{r}^{M}\right)>M n .
$$

Let $\bar{r}$ denote the image of $r$ in $g r R_{q(r)}$. If $\beta(\bar{r})=0$, then $\bar{q}(r)>q(r)$, so $\exists M>0, q\left(r^{M}\right)>M q(r)$. Hence $(\bar{r})^{M}=0$. So the kernel is exactly the nilpotents. 
If gr $R$ has no nilpotents, then there does not exist $\bar{r} \in \operatorname{gr} R_{n} \backslash\{0\}$ with $\bar{r}^{M}=0$. So $q\left(r^{M}\right)$ is not more than $M n$; indeed $q\left(r^{M}\right)=M n$ for all $M$. Hence $\bar{q}(r)=n$, and $\overline{\mathrm{q}}=\mathrm{q}$. Thus $\overline{\mathrm{gr}}=$ gr naturally.

Since $\overline{g r} \mathrm{R}$ has no nilpotents, gr R can only be isomorphic to it if it too has no nilpotents.

We now switch over to the geometric point of view, in which we map from the balanced normal cone to the ordinary one. The above proposition tells us that the map $\overline{\mathrm{C}}_{W} V \rightarrow \mathrm{C}_{W} V$ factors as $\overline{\mathrm{C}}_{W} V \rightarrow\left(\mathrm{C}_{W} V\right)_{\text {red }} \hookrightarrow \mathrm{C}_{W} V$, where $\left(\mathrm{C}_{W} V\right)_{\text {red }}$ denotes the reduction of $C_{W} V$. So $\left(C_{W} V\right)_{\text {red }}$ serves as an intermediary when trying to compare the spaces $C_{W} V$ and $\bar{C}_{W V} V$. This motivates our looking at Chow groups, since $A_{\bullet}\left(C_{W} V\right)=A_{\bullet}\left(\left(C_{W} V\right)_{\text {red }}\right)$.

Theorem 1. Let $\mathrm{W}$ be a closed subscheme of $\mathrm{V}$, where $\mathrm{V}$ is reduced. The induced map $\beta: \overline{\mathrm{C}}_{W} \mathrm{~V} \rightarrow \mathrm{C}_{W} \mathrm{~V}$ is proper, with finite fibers. Assume now that $\mathrm{V}$ is of finite type over a field. Then the two maps $\overline{\mathrm{C}}_{W} \mathrm{~V} \rightarrow\left(\mathrm{C}_{W} \mathrm{~V}\right)_{\text {red }},\left(\mathrm{C}_{W} \mathrm{~V}\right)_{\text {red }} \hookrightarrow \mathrm{C}_{W} \mathrm{~V}$ induce the same Chow class in $\mathrm{A} \cdot\left(\left(\mathrm{C}_{W} \mathrm{~V}\right)_{\text {red }}\right)$.

(We expect that the field hypothesis on $\mathrm{V}$ is largely unnecessary; it is used to ensure that some normalizations are finite.)

Note that these two Chow classes are induced on $\left(\mathrm{C}_{W V} V\right)_{\text {red }}$ in very different ways, as we go over in section 6 . The inclusion $\left(\mathrm{C}_{W} V\right)_{\text {red }} \hookrightarrow \mathrm{C}_{W} V$ defines a class by taking the sum of the top-dimensional components weighted by the lengths of the local rings on the target. Whereas the surjection $\bar{C}_{W} V \rightarrow\left(C_{W} V\right)_{\text {red }}$ defines a class by taking the sum of the top-dimensional components weighted by the degree of the map over those components.

Example 2. Let $\mathrm{V}$ be the line with coordinate $\mathrm{x}$, and $\mathrm{W}$ the doubled origin (defined by $x^{2}=0$ ). Then $C_{W} V$ is the doubled line, whereas $\bar{C}_{W} V$ is just the ordinary line; see example 1 for these calculations. The map $\overline{\mathrm{C}}_{W} \mathrm{~V} \rightarrow\left(\mathrm{C}_{W} \mathrm{~V}\right)_{\text {red }} \hookrightarrow \mathrm{C}_{W} \mathrm{~V}$ is the squaring map from the line to the (reduction of the doubled) line.

It can happen that $\bar{C}_{W} V$ has more components than $C_{W} V$, not because a component collapses (since we know there are finite fibers), but because several components of $\bar{C}_{W} V$ cover the same component of $C_{W} V$. When this happens, we get a refinement of the multiplicities in the fundamental class of $C_{W} V$; the multiplicity of a component $\mathrm{F} \subseteq \mathrm{C}_{W} \mathrm{~V}$ is the sum over those components $\overline{\mathrm{F}} \subseteq \overline{\mathrm{C}}_{W} \mathrm{~V}$ whose image is $F$, of the degree of the $\operatorname{map} \bar{F} \rightarrow F$.

Example 3. Let $\mathrm{R}=\mathbb{F}[\mathrm{a}, \mathrm{b}] /\left\langle\mathrm{a}^{2}-\mathrm{b}^{2}\right\rangle$, so $\mathrm{V}:=\operatorname{Spec} \mathrm{R}$ is the union of two lines. Let $I=\langle b\rangle$, so $W$ is a double point at the origin. $C_{W} V$ is a trivial line bundle over $W, q(a)=0, \bar{q}(a)=1$, and $\overline{g r} R \cong R$. The map $\overline{\mathrm{C}}_{W} \mathrm{~V} \rightarrow \mathrm{C}_{W} V$ maps the two lines onto the reduction of $\mathrm{C}_{W} \mathrm{~V}$. 
In this way, the fundamental class of $\mathrm{C}_{W} \mathrm{~V}$ is a sum of the two (equal)

Chow classes induced by the lines in $\overline{\mathrm{C}}_{W} V$.

To prove theorem 1, we need a number of basic results about balanced normal cones, which will come in section 2 . The proof itself will come in section 6 ; essentially, what is shown is that the sheaves associated to $\overline{\mathrm{C}}_{W} V$ and $\mathrm{C}_{W} V$ define the same element in K-homology (the Grothendieck group of formal differences of coherent sheaves, not just vector bundles) of a certain thickening of $\mathrm{C}_{W} \mathrm{~V}$, and this is enough. (An earlier version of this paper had a much more difficult argument based on blowups.)

\subsection{The "basic construction" in intersection theory. We recall the basic construction from [FM].}

Let $\mathrm{X} \hookrightarrow \mathrm{Y}$ be an inclusion (soon, a regular embedding), and $\mathrm{V} \rightarrow \mathrm{Y}$ a morphism. Let $W$ be the pullback, so we have a square

$$
\begin{aligned}
& W \hookrightarrow \mathrm{V} \\
& \downarrow \\
& \mathrm{X} \hookrightarrow \mathrm{Y}
\end{aligned}
$$

Now replace each of the big schemes ( $\mathrm{Y}$ and $\mathrm{V}$ ) by the normal cones to the subschemes. This allows us to reverse the horizontal arrows, replacing inclusions by epimorphisms.

$$
\begin{aligned}
& W \leftarrow C_{W V} V \\
& \downarrow \\
& X \leftarrow C_{X} Y
\end{aligned}
$$

This is no longer a pullback diagram; we only have a map from $C_{W} V$ to the actual pullback $N$. Hence $C_{W} V$ defines a Chow class on $N$. While it is not hard to check that the map $C_{W} V \rightarrow N$ is an inclusion, this property doesn't seem to play any role in the construction, and will not hold in our variation below.

For purposes of intersection theory, it turns out to be useful to require that $\mathrm{X} \hookrightarrow \mathrm{Y}$ be a regular embedding, i.e. that $\mathrm{C}_{\mathrm{X}} \mathrm{Y}$ be a vector bundle. This is because Fulton and MacPherson's goal is to define a Chow class down on W (not up on $\mathrm{N}$ ), which they call the "refined intersection product" of $X$ and $V$. (It can be thought of as a cap product, where the regular embedding $X \hookrightarrow Y$ plays the role of the cobordism class and the map $\mathrm{V} \rightarrow \mathrm{Y}$ that of the bordism class.) This is done using a Thom-Gysin isomorphism $A_{\bullet}(N) \cong A_{\bullet-d}(W)$, which holds if $N$ is a vector bundle of some dimension $d$. That in turn is guaranteed if $C_{X} Y$ is a vector bundle, motivating that condition. This completes the basic construction.

How do things change in what we will call the balanced basic construction, where we instead use balanced normal cones? 
First, we will require that $\mathrm{V}$ is reduced, in order to apply theorem 1 . This is not a particularly stringent assumption from the point of view of intersection theory, where any Chow class of a nonreduced subscheme is naturally a sum of classes of reduced subschemes.

As before, by passing to the cones we can reverse the horizontal arrows. However, these reversed maps are no longer epimorphisms - they only hit the reductions (thanks to the last part of proposition 1).

$$
\begin{aligned}
& W \hookleftarrow W_{\text {red }} \leftarrow \bar{C}_{W V} V \\
& \downarrow \\
& X \hookleftarrow X_{\text {red }} \leftarrow \overline{\mathrm{C}}_{X}^{\downarrow} Y
\end{aligned}
$$

If we assume that $X$ is smooth and $X \hookrightarrow Y$ is a regular embedding, then $C_{X} Y$ is reduced (as it is a vector bundle over something reduced). Hence $\bar{C}_{X} Y=C_{X} Y$, and the pullback to $\mathrm{W}$ is again $\mathrm{N}$.

However, even in this case, $W$ and $C_{W} V$ are typically not reduced. So $\bar{C}_{W} V$, which $i s$ reduced, is something new. It too maps (though usually not injectively) to the pullback bundle $\mathrm{N}$, and this map factors as $\overline{\mathrm{C}}_{W} \mathrm{~V} \rightarrow\left(\mathrm{C}_{W} \mathrm{~V}\right)_{\text {red }} \hookrightarrow \mathrm{N}_{\text {red }} \hookrightarrow \mathrm{N}$.

Theorem 2. Assume that $\mathrm{X} \hookrightarrow \mathrm{Y}$ is a regular embedding, and $\mathrm{V} \rightarrow \mathrm{Y}$ a morphism, with $\mathrm{V}$ reduced. Let $\mathrm{W}$ be the pullback of $\mathrm{X}, \mathrm{V} \rightarrow \mathrm{Y}$.

Then Fulton and MacPherson's refined intersection product $\mathrm{X} \cdot \mathrm{V} \in \mathrm{A} \bullet(\mathrm{W})$, usually calculated with $\mathrm{C}_{W} \mathrm{~V}$, can be calculated equally well with $\overline{\mathrm{C}}_{W} \mathrm{~V}$.

Proof. Since the map $\overline{\mathrm{C}}_{W} \mathrm{~V} \rightarrow \mathrm{N}$ factors through $\beta: \overline{\mathrm{C}}_{W} \mathrm{~V} \rightarrow \mathrm{C}_{W} \mathrm{~V}$, theorem 1 implies that $\overline{\mathrm{C}}_{W} V$ and $\mathrm{C}_{W} V$ induce the same Chow class on $\mathrm{N}$.

Example 4. Let $\mathrm{Y}, \mathrm{V}$ be affine lines with coordinates $\mathrm{y}, v$, let $\mathrm{X}$ be the origin in $Y$, and let $V \rightarrow Y$ be the squaring map $y=v^{2}$. Then $W$ is the doubled origin in $\mathrm{V}$, defined by $v^{2}=0$.

In ordinary intersection theory, the normal cones $C_{W} V, C_{X} Y$ and the pullback $N$ are all trivial line bundles, over $W, X, W$ respectively. The map $\mathrm{C}_{W} \mathrm{~V} \rightarrow \mathrm{N}$ is an isomorphism, inducing the fundamental class on $\mathrm{W}$, which is the twice the class of the reduced point $W_{\text {red }}$.

In the balanced basic construction, the balanced normal cone $\bar{C}_{W} V$ is the trivial line bundle over $W_{\text {red }}$, and the map $\bar{C}_{W} V \rightarrow N$ is the squaring map, rather than an isomorphism. One calculates this on the algebra side, where the diagram above is

$$
\begin{array}{rlrl}
\mathbb{F}[v] /\left\langle v^{2}\right\rangle & \rightarrow \mathbb{F} \hookrightarrow \mathbb{F}\left[v_{(1 / 2)}\right] \\
\uparrow & \uparrow & \uparrow \\
\mathbb{F} & =\mathbb{F} \hookrightarrow \mathbb{F}\left[\mathbf{y}_{(1)}\right] .
\end{array}
$$

Here the parenthesized subscripts indicate the degree in these graded rings. In the graded map on the right, $y \mapsto v^{2}$. The pushout Fun $\left(\mathrm{N}_{\text {red }}\right)$ of that 
right square is obviously $\mathbb{F}[y]$, so this squaring map is the one $\bar{C}_{W} V \rightarrow N_{\text {red }}$ claimed above, inducing twice the fundamental Chow class of $\mathrm{N}_{\text {red }}$. The Gysin map then takes that to twice the fundamental class of the reduced point $W_{\text {red }}$, as predicted by theorem 2 .

Because the space $\bar{C}_{W} V$ can have more components than $C_{W} V$, as in example 3, we can refine Fulton and MacPherson's "refined intersection products" further as a sum over the components of $\overline{\mathrm{C}}_{W} \mathrm{~V}$.

Example 5. The further refinement in example 3 only reflected the fact that $\mathrm{V}$ itself was reducible. The example below, which we shall compute in section 3 , shows the refinement can be nontrivial even when $\mathrm{V}$ is irreducible.

Let $Y=\operatorname{Spec} \mathbb{F}[a, b], X$ the $a$-axis, and $V$ the nodal cubic curve $b^{2}=a^{2}(a+1)$. Their intersection $\mathrm{X} \cap \mathrm{V}=: \mathrm{W}$ is a double point at the origin (the node of the cubic) and a reduced point at $(-1,0)$. The map from $\mathrm{C}_{W} \mathrm{~V}$ to the pullback $W \times \mathbb{A}^{1}$ of the (trivial) normal bundle $\mathrm{C}_{\mathrm{X}} \mathrm{Y}$ is an isomorphism, inducing the fundamental Chow class on $W \times \mathbb{A}^{1}$ and

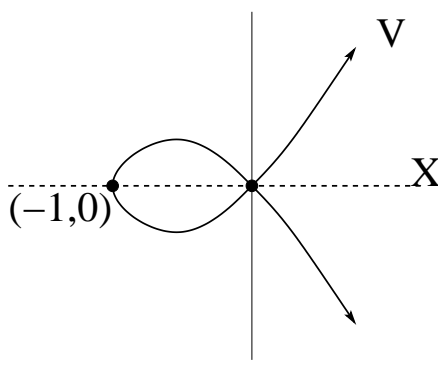
thereby on $W$.

In this case, the Chow ring calculation (on the projective plane) gives 3 times the class of a point. The refined intersection product just calculated splits this as $3=2+1$, from the double point and single point.

To compute the balanced normal cone $\bar{C}_{W} V$, present $V=$ Spec $R$ using $R=\mathbb{F}[a, b, c] /\left\langle c-a(a+1), b^{2}-a c, b^{2}(a+1)-c^{2}\right\rangle$. Then $c^{2} \in\langle b\rangle^{2}$, so $\bar{q}(c)$ is plainly at least 1 . As we will be able to compute later (using proposition 4), in fact $\overline{\mathbf{q}}(\mathrm{a})=0, \overline{\mathrm{q}}(\mathrm{c})=1$, and

$$
\begin{aligned}
\overline{\operatorname{gr}} R & =\mathbb{F}[a, b, c] /\left\langle a(a+1), a c, b^{2}(a+1)-c^{2}\right\rangle \\
& =\mathbb{F}[a, b, c] /(\langle a+1, c\rangle \cap\langle a, b-c\rangle \cap\langle a, b+c\rangle) .
\end{aligned}
$$

So $\bar{C}_{Y} X$ is an isolated line union a pair of intersecting lines, and the sum of these components further refines the intersection calculation as $3=$ $1+(1+1)$.

Unlike $R$ and gr $R$, this ring $\overline{\text { gr }} \mathrm{R}$ is not generated over $\mathbb{F}$ by two elements.

It would be interesting to find the branch locus of the map $\bar{C}_{W} V \rightarrow\left(C_{W} V\right)_{\text {red }}$ in genuine intersection theory examples, and see what that and more refined degeneracy loci mean for enumerative questions. It would also be interesting to see how the monodromy group of the branched cover relates to the "Galois group" of the enumerative problem $[\mathrm{Hr}]$. 
We now outline the rest of the paper. In section 2 we describe Rees' formula for $\bar{q}$ and give the basic results about gr $R$. When gr $R$ is reduced, then $\overline{g r} R=g r R$; we present a number of examples to show some possible reasons that $\mathrm{gr} R \neq$ gr $R$. In section 3 we study the intersection of a variety in affine space with a hyperplane, and geometrically describe the normal cone (and under certain conditions, the balanced normal cone) as flat limits. In section 4 we introduce the ring $\widetilde{\text { gr }} \mathrm{R}$ with which to further study $\overline{g r} R$ in the case that $I$ is principal, and we compute several examples. Finally, in section 6 we prove theorem 1.

1.4. Acknowledgements. It is a pleasure to thank Valery Alexeev, Tom Graber, Mark Gross, Joseph Gubeladze, Craig Huneke, Bernd Sturmfels, and Ravi Vakil. The anonymous referee was extremely helpful and their input greatly improved this paper. I give special thanks to Bernard Teissier for sharing with me his unpublished manuscript [LJT].

Many examples in this paper were worked out with the help of the computer algebra system Macaulay 2 [M2].

\section{Properties of $\overline{g r}$}

2.1. Rees' formula for $\bar{q}$. In this section $\mathrm{R}$ is a ring without nilpotents. (And commutative, Noetherian, and with unit, as per our standing assumptions.)

Assume to begin with that $R$ is an integrally closed domain, and $I=\langle b\rangle$ is a principal ideal for $b$ a nonzerodivisor. Let $D_{1}, \ldots, D_{k}$ be the components of I's vanishing set, and $v_{i}$ the corresponding valuations.

Then for all $\mathrm{n}$, we have $\mathrm{q}(\mathrm{r}) \geq \mathrm{n} \Longleftrightarrow \mathrm{r} \in\left\langle\mathrm{b}^{\mathfrak{n}}\right\rangle \Longrightarrow v_{\mathfrak{i}}(\mathrm{r}) \geq \mathfrak{n} v_{\mathrm{i}}(\mathrm{b})$; contrapositively,

$$
q(r) \leq \min _{i} \frac{v_{i}(r)}{v_{i}(b)}
$$

The same bound follows for $\bar{q}$. Rees' theorem, in this special case, says that $\bar{q}$ is actually given by this formula.

Example 6. Let $\mathrm{P}$ be a lattice polytope, and $\mathrm{R}$ the homogeneous coordinate ring of the projective toric variety $X=X_{P}$, which has a basis given by lattice points in dilations of $\mathrm{P}$. Let $\mathrm{b}$ be the degree 1 element corresponding to some lattice point $p \in P$. Then the valuations $\left\{v_{i}\right\}$ in the formula for $\bar{q}$ correspond to the facets of $\mathrm{P}$ not containing $\mathrm{p}$. If $\mathrm{o}$ is any lattice point in $P$ and $r$ the corresponding ring element, then $v_{i}(r)$ is the distance of o to the $i$ facet, measured in lattice units.

Let $f: P \rightarrow \mathbb{R}$ denote the continuous piecewise-linear function measuring the distance of $\mathrm{q}$ to a far wall of $\mathrm{P}$ along the straight line connecting $\mathrm{p}$ and $q$; it takes the value 1 at $p, 0$ on all facets $F$ not containing $p$, and varies linearly on the cone from $p$ to $F$. Then if $r \in R$ is a basis element 
corresponding to a lattice point $p \in P$, we have $\bar{q}(r)=f(p)$. (More generally, if $r \in R$ is a basis element corresponding to a lattice point $o$ in the $k$-fold dilation $k P$ of $P$, we have $\bar{q}(r)=k f(o / k)$.) An example is in figure 1 .

The ring $\overline{g r} \mathrm{R}$ is the homogeneous coordinate ring of a union of projective toric varieties, whose components are (weighted) cones on the facets of $P$ not containing $p$. This reducibility arises from the fact that in the associated graded, the product of two basis elements can be zero, which happens if and only if when projecting away from $p$ the corresponding points in $\mathrm{P}$ do not project to a common facet.

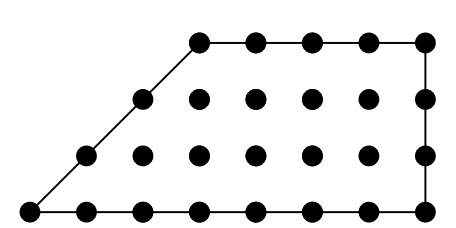

$\mathrm{p}$

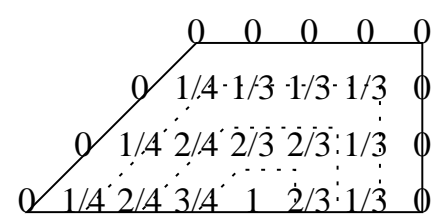

$\mathrm{p}$

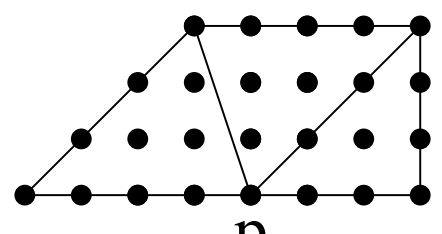

$\mathrm{p}$

Figure 1. A lattice polytope, the valuation $\overline{\mathbf{q}}$ (and its level sets) evaluated on the generators, and the polyhedral complex arising from $\overline{g r} R$.

More general reduced torus-equivariant degenerations of toric varieties were studied in $[\mathrm{Al}]$.

If $R$ is not integrally closed, the valuations $\left\{v_{i}\right\}$ may not be simply associated to divisors. For example, let $R=\mathbb{F}[x, y] /\langle x y\rangle$, and $I=\left\langle x+y^{2}\right\rangle$. Then I vanishes at the origin, to order 1 if one approaches along the $x$-axis and order 2 if one approaches along the $y$-axis. Hence "the order of vanishing at the origin" is not well-defined. But the same formula can be made to work by restricting divisorial valuations from the integral closure of $R$ in $R\left[b^{-1}\right]$. (This integral closure will show up in a different guise in section 4.)

It is easy to generalize to the case $\mathrm{I}=\langle\mathrm{b}\rangle$ for $\mathrm{b}$ a zero divisor:

$$
\bar{q}(r)= \begin{cases}0 & \text { if } r \text { doesn't vanish on all the components that } b \text { does } \\ \min _{i} \frac{v_{i}(r)}{v_{i}(b)} & \text { if } r \text { does vanish on them }\end{cases}
$$

where the valuations are pulled back from the quotient ring $R / \operatorname{ann}(b)$.

There is a further generalization of this formula, which we won't need, to the case that I is not principal; it uses the blowup algebra to reduce to the principal case. One interesting aspect of this is that if Spec R or Spec R/I are singular along Spec R/I, then the exceptional locus in the blowup may have more components than Spec R/I does itself, and we need them for Rees' formula. 
Lemma 1 (Samuel). Let $\mathrm{R}=\oplus_{\mathrm{n} \in \mathbb{N}} \mathrm{R}_{\mathrm{n}}$ be a graded Noetherian ring. Then $\mathrm{R}$ is finitely generated as an algebra over $\mathrm{R}_{0}$.

Proof. Let $r_{1}, \ldots, r_{k}$ be homogeneous generators of the augmentation ideal $R_{+}$. Let $r$ be a homogeneous element of positive degree. Then $r=\sum_{i} c_{i} r_{i}$ for some homogeneous $\left(c_{i}\right)$ with $\operatorname{deg} c_{i}<\operatorname{deg} r$. By induction, these $c_{i}$ are polynomials in the $\left(r_{j}\right)$ with coefficients from $R_{0}$. Hence $r$ is such a polynomial too.

By the direct sum assumption, every element of $R$ is a sum of such rs and an element of $R_{0}$.

Proposition 3. Let $\mathrm{I}=\langle\mathrm{b}\rangle$ be a principal ideal in a ring $\mathrm{R}$.

- The multiplication map $\mathrm{b} \cdot: \operatorname{gr} \mathrm{R}_{\mathrm{i}} \rightarrow \operatorname{gr} \mathrm{R}_{\mathrm{i}+1}$ is always onto.

- The multiplication map $\mathrm{b} \cdot: \operatorname{gr} \mathrm{R}_{\mathbf{i}} \rightarrow$ gr $\mathrm{R}_{\mathbf{i}+1}$ is $1: 1$ for all large $i$.

- The multiplication map $\mathrm{b} \cdot: \overline{g r} \mathrm{R}_{\mathfrak{i}} \rightarrow \overline{\text { gr }} \mathrm{R}_{\mathfrak{i}+1}$ is always $1: 1$ for $\mathrm{i}>0$. If $\mathrm{b}$ is not a zero divisor in $\mathrm{R}$, then $\mathrm{b} \cdot: \overline{\mathrm{gr}} \mathrm{R}_{0} \rightarrow \overline{\mathrm{gr}} \mathrm{R}_{1}$ is also $1: 1$.

- The multiplication map $\mathrm{b} \cdot: \overline{\mathrm{gr}} \mathrm{R}_{\mathrm{i}} \rightarrow \overline{\mathrm{gr}} \mathrm{R}_{\mathrm{i}+1}$ is onto for all large $\mathrm{i}$.

Note that the first two claims are only interesting for $i \in \mathbb{N}$, whereas the second two are interesting for $i \in \mathbb{Q}$.

Proof. The first claim is essentially tautological.

For the second, consider the ascending chain $\operatorname{ann}(b) \leq \operatorname{ann}\left(b^{2}\right) \leq \operatorname{ann}\left(b^{3}\right) \leq$ $\ldots$ of annihilator ideals in gr R. Let $j$ be the stage at which it stabilizes. Then for $\bar{c} \in$ gr $R, b^{j} c \neq 0$ implies $b^{k} c \neq 0$ for any $k \geq j$. With this and the first claim, we see that if $d \in \operatorname{gr} R_{k} \backslash\{0\}, k \geq j$, then $b d \neq 0$.

The third and fourth don't depend on $R$ as much as $R$ mod its nilpotents, so we assume now that $\mathrm{R}$ has none.

For the third, let $c \in R$. If $b$ vanishes on a component of Spec $R$ on which $c$ doesn't, then (1) b is a zero divisor and (2) $\bar{q}(c)=0$. So now we assume that $c$ vanishes on each component of Spec $R$ on which $b$ vanishes, and we can pass to $\mathrm{R} / \mathrm{ann}(\mathrm{b})$.

Now, $\bar{q}(c)=\min _{i} \frac{v_{i}(c)}{v_{i}(b)}$. Then

$\bar{q}(b c)=\min _{i} \frac{v_{i}(b c)}{v_{i}(b)}=\min _{i} \frac{v_{i}(b)+v_{i}(c)}{v_{i}(b)}=\min _{i} 1+\frac{v_{i}(c)}{v_{i}(b)}=1+\min _{i} \frac{v_{i}(c)}{v_{i}(b)}=1+\bar{q}(c)$.

So $c \neq 0$ implies $\overline{\operatorname{gr}}(\mathrm{bc}) \neq 0$. Contrapositively, the only way for $\overline{\mathrm{gr}}(\mathrm{c} \neq 0)$ to be annihilated by $\mathrm{b}$. is for $\mathrm{b}$ to be a zero divisor and for $\overline{\mathrm{q}}(\mathrm{c})=0$. This gives the third claim.

For the last claim, since $\overline{\text { gr }} \mathrm{R}$ is Noetherian, let $\overline{\mathrm{g}}_{1}, \ldots, \overline{\mathrm{g}}_{\mathrm{G}}$ generate $\overline{\mathrm{gr}} \mathrm{R}$ as an algebra over gr $R_{0}$ (using lemma 1 ). Then any monomial in the $\left\{\bar{g}_{i}\right\}$ of high degree 
must involve some $\bar{g}_{i}$ to a high power. Since each $g_{i} \in \sqrt{\langle b\rangle}$, having $\bar{g}_{i}$ to a high power means that a factor of $\bar{b}$ can be extracted. This establishes the fourth claim.

The following gives a characterization of $\bar{q}$ that is useful for verifying examples, and in section 3 will also be of use in interpreting balanced normal cones geometrically. It uses the concept of homogeneous filtrations $p$, meaning $p\left(r^{\mathfrak{n}}\right)=n p(r)$ $\forall \mathrm{r} \in \mathrm{R}, \mathrm{n} \in \mathbb{N}$.

Proposition 4. The filtration $\overline{\mathrm{q}}$ is the unique minimum homogeneous filtration $\mathrm{p}$ with $\mathrm{p}(\mathrm{b})=1$. In other words, let $\mathrm{p}$ be a homogeneous filtration on $\mathrm{R}$ such that $\mathrm{p}(\mathrm{b})=1$. Then $\mathrm{p}(\mathrm{r}) \geq \overline{\mathrm{q}}(\mathrm{r}) \forall \mathrm{r} \in \mathrm{R}$.

If $\mathrm{R}=\mathbb{F}\left[\mathrm{a}_{1}, \ldots, \mathrm{a}_{\mathrm{n}}, \mathrm{b}\right] / \mathrm{J}$, and $w_{1}, \ldots, w_{\mathrm{n}} \geq 0$ are lower bounds on $\overline{\mathrm{q}}\left(\mathrm{a}_{1}\right), \ldots$, $\bar{q}\left(a_{n}\right)$, then let $\mathrm{p}$ be the (possibly inhomogeneous) filtration induced on $\mathrm{R}$ from the filtration $\mathrm{p}\left(\mathrm{b}^{\mathrm{B}} \prod_{i} \mathrm{a}_{i}^{n_{i}}\right)=\mathrm{B}+\sum_{i} n_{i} \mathcal{w}_{i}$ on the polynomial ring. If the associated graded to $\mathrm{p}$ has no nilpotents, then $\mathrm{p}=\overline{\mathrm{q}}$.

Proof. By the existence of the limit $\bar{q}$, given $r \in R, \epsilon>0$, for all large $n$ we have $\mathrm{q}\left(\mathrm{r}^{\mathrm{n}}\right) / \mathrm{n} \geq \overline{\mathrm{q}}(\mathrm{r})-\epsilon$. Hence $\mathrm{r}^{\mathrm{n}}=a \mathrm{~b}^{\lfloor n(\overline{\mathrm{q}}(\mathrm{r})-\epsilon)\rfloor}$ for some $\mathrm{a} \in \mathrm{R}$. Then

$$
\begin{aligned}
p(r) & =\frac{1}{n} p\left(r^{n}\right)=\frac{1}{n} p\left(a b^{\lfloor n(\bar{q}(r)-\epsilon)\rfloor}\right) \geq \frac{1}{n} p\left(b^{\lfloor n(\bar{q}(r)-\epsilon)\rfloor}\right) \\
& =\frac{1}{n}\lfloor n(\bar{q}(r)-\epsilon)\rfloor \geq \frac{1}{n}(n(\bar{q}(r)-\epsilon)-1)=\bar{q}(r)-\epsilon-\frac{1}{n}
\end{aligned}
$$

hence $p(r) \geq \bar{q}(r)$.

For the second part, saying that the associated graded to $p$ has no nilpotents is the same as saying that $p$ is homogeneous. Plainly $p(b)=1$. So by the first part, $p \geq \bar{q}$. Since $p$ is the smallest filtration with $p\left(a_{i}\right)=w_{i}$, and $w_{i} \leq \bar{q}\left(a_{i}\right)$ by assumption, we have $p \ngtr \bar{q}$. So $p=\bar{q}$.

Note that not every homogeneous filtration on a polynomial ring mod an ideal is of the form in the second part of the proposition - for example, the $\langle x+y\rangle$-adic filtration on $\mathbb{F}[x, y]$. We will only be able to apply the second part of proposition 4 when the generating set has been chosen felicitously.

In some of the examples to come, we will present $R$ as a polynomial ring modulo an ideal. We'll determine some lower bounds $\left\{\boldsymbol{w}_{i}\right\}$ on the $\overline{\mathrm{q}} \mathrm{s}$ of the variables, including $\bar{q}(b)=1$, and consider the induced (a priori inhomogeneous) filtration $p$. To compute the associated graded to $p$, we check that the generating set of the ideal is a Gröbner basis with respect to some term order respecting this weighting of the variables, and replace each relation by its lowest-weight component. To be sure we're satisfying proposition 4 , it remains to check that the associated graded has no nilpotents. When all goes well and that turns out to be true, we 
learn three things: $p=\bar{q}$, our lower bounds $\left\{w_{i}\right\}$ were correct, and each filtered piece of $R$ intersected with the linear span of the variables is spanned by a subset thereof.

2.2. Examples. Here are some of the nonobvious possible behaviors of $\bar{q}$ and $\overline{\mathrm{gr}}$.

2.2.1. The limit $\overline{\mathbf{q}}$ need not be achieved. One way of thinking about the limit $\lim _{\mathfrak{n} \rightarrow \infty} \frac{\mathrm{q}\left(\mathrm{r}^{\mathfrak{n}}\right)}{\mathrm{n}}$ is to take the limit through a subsequence $1=\mathrm{n}_{1}\left|\mathrm{n}_{2}\right| \mathrm{n}_{3} \mid \cdots$, which is easily seen to be increasing:

$$
q(r)=\frac{q\left(r^{n_{1}}\right)}{n_{1}} \leq \frac{q\left(r^{n_{2}}\right)}{n_{2}} \leq \frac{q\left(r^{n_{3}}\right)}{n_{3}} \leq \ldots \leq \bar{q}(r) .
$$

Many people's first guess, upon learning the definition of $\bar{q}$, is that the limit $\bar{q}(r)$ is achieved for some finite $n$. This turns out to be true if $R$ is integrally closed.

Proposition 5 (Rees). Let $\mathrm{R}$ be an integrally closed domain, and $\mathrm{I}=\langle\mathrm{b}\rangle$. Then there exists $\mathrm{N}>0$ such that $\overline{\mathrm{q}}(\mathrm{r})=\frac{1}{\mathrm{~N}} \mathrm{q}\left(\mathrm{r}^{\mathrm{N}}\right)$.

Proof. Let $\mathrm{N}$ be the least common multiple of the valuations $v_{\mathfrak{i}}(\mathrm{b})$, so $\mathrm{N} \overline{\mathrm{q}}$ is $\mathbb{N}$-valued. Then for any $r$, the rational function $r^{N} / b^{N \bar{q}(r)}$ satisfies the valuative criterion for integrality. (We asked that $\mathrm{R}$ be a domain so that $\mathrm{b}$ is not a zero divisor.) Since $R$ is integrally closed, $r^{N} / b^{N \bar{q}(r)}=s$ for some $s \in R$. Hence $q\left(r^{N}\right) \geq N \bar{q}(r)$, but we already knew the opposite inequality.

Example 7. This is a variant of example 3, with the same geometry.

Let $R=\mathbb{F}[a, b] /\left\langle a^{2}-a b\right\rangle$, and $I=\langle b\rangle$. Then $a^{n}=a b^{n-1}$, and in fact $q\left(a^{\mathfrak{n}}\right)=n-1$. Taking the limit, $\bar{q}(a)=1$. But for no $n$ is $q\left(a^{\mathfrak{n}}\right) / \mathfrak{n}=\bar{q}(a)$.

2.2.2. $\overline{g r} \mathrm{R} \neq \operatorname{gr} \mathrm{R}$ despite being integer-graded. We've already shown that $\mathrm{q}=\overline{\mathrm{q}}$, if and only if gr $R$ has no nilpotents, if and only if $\overline{g r} R \cong \operatorname{gr} R$. One obvious reason for $\overline{g r} R$ to be different from gr $R$ is if $v_{i}(b)>1$ for some valuation $v_{i}$ in Rees' formula, and $\overline{g r} \mathrm{R}$ to have support in other than integer degrees. Geometrically, this corresponds to the divisor $b=0$ not being generically reduced. (It is still possible for $\overline{\mathrm{gr}} \mathrm{R}$ to be integer-graded, as example 3 shows.)

This raises the question: if the divisor $b=0$ is generically reduced, does that force $\overline{\operatorname{gr}} \mathrm{R}=\mathrm{gr} R$ ? To construct a counterexample, it will suffice to make the divisor generically reduced but not reduced, hence not satisfying Serre's criterion S1. So the ambient Spec R shouldn't satisfy Serre's criterion S2, the canonical example being the union of two planes in 4-space.

Let $R=\mathbb{F}[b, c, d, e] /\langle d(b-d), d c, e(b-d), e c\rangle$, the union of the $d=e=0$ plane and $b-d=c=0$ plane. Then the $b$-divisor is $\operatorname{Spec} \mathbb{F}[b, c, d, e] /\left\langle b, d^{2}, d c, e d, e c\right\rangle$, 
supported on the $\mathrm{b}=\mathrm{d}=\mathrm{e}=0$ line union the $\mathrm{b}=\mathrm{d}=\mathrm{c}=0$ line, with an extra point embedded at the origin.

Since $d^{N}=d b^{N-1}$ for all $N>1$, we see $\bar{q}(d) \geq \frac{N-1}{N}$. So $\bar{q}(d) \geq 1$, and the lower bounds $\left\{w_{i}\right\}$ we can guess for the $\bar{q}$ of the variables are $\bar{q}(b), \bar{q}(d) \geq 1$, $\bar{q}(c), \bar{q}(e) \geq 0$. (Note that $\bar{q}(d) \neq q(d)=0$.)

The relations are homogeneous with respect to this weighting, hence the associated graded gr $\mathrm{R}$ turns out to be isomorphic to $\mathrm{R}$ :

$$
\operatorname{gr} R=\mathbb{F}\left[b_{(1)}, c, d_{(1)}, e\right] /\langle e c, d(b-d), d c, e(b-d)\rangle .
$$

Since this has no nilpotents, we can use proposition 4 to know that we have correctly calculated $\bar{q}$. (Side note: the fact that $\overline{g r} R \cong R$ doesn't mean that $\overline{g r} R$ is boring - rather, it has served as a means of discovering a grading with which to better understand $\mathrm{R}$ itself.)

Whereas gr $R=\mathbb{F}\left[b_{(1)}, c, d, e\right] /\left\langle e c, d^{2}, d c, e d\right\rangle$, whose quotient by $\sqrt{0}=\langle d\rangle$ is $\mathbb{F}\left[b_{(1)}, c, e\right] /\langle e c\rangle$. Geometrically, the map gr $R \rightarrow \operatorname{gr} R /\langle d\rangle \hookrightarrow \operatorname{gr} R$ corresponds (in reverse) to a pair of planes meeting at a point, mapping onto a pair of planes meeting along a line, mapping into a thickening of that scheme along the line.

2.2.3. $\overline{\mathrm{gr}} \mathrm{R} \neq \mathrm{gr} \mathrm{R}$ despite the divisor being reduced. It is curious that this can only happen if $b$ is a zero divisor, as we now prove.

Proposition 6. Let the ring $\mathrm{R}$ contain the element $\mathrm{b}$, and assume $\mathrm{R} /\langle\mathrm{b}\rangle=\operatorname{gr} \mathrm{R}_{0}$ has no nilpotents. If $\mathrm{b}$ is not a zero divisor, then gr $\mathrm{R}$ also has no nilpotents, so $\overline{\mathrm{q}}=\mathrm{q}$ and $\overline{\mathrm{gr}} \mathrm{R}=\mathrm{gr} \mathrm{R}$.

Proof. Assume $c \in R$ is nonzero, and $q(c)=n>0$, so $c$ has image $\dot{c} \in \operatorname{gr} R_{n}$. Assume also that $\dot{c}^{m}=0$, so $q\left(c^{m}\right)>m n$.

Then we can write $c=a b^{n}$ and $c^{m}=d b^{m n+1}$, where $q(a)=0$. So $c^{m}=$ $a^{m} b^{m n}=d b^{m n+1}$, hence $b^{m n}\left(a^{m}-b d\right)=0$. Since $b$ is not a zero divisor, $a^{m}-$ $\mathrm{bd}=0$, so $\mathrm{q}\left(\mathrm{a}^{\mathrm{m}}\right) \geq 1$. Hence $\dot{\mathrm{a}}$ is a nilpotent element of gr $\mathrm{R}_{0}$, contradiction.

To find an example in which gr $\mathrm{R}$ has nilpotents, but only after degree 0 , we therefore need to allow $b$ to be a zero divisor. The proof above suggests ${ }^{1}$ $\mathrm{I}=\left\langle\mathrm{c}-\mathrm{ab}, \mathrm{c}^{2}-\mathrm{db}^{4}\right\rangle$, which is almost good enough, we just need to take its radical (using [M2]):

$I=\sqrt{\left\langle c-a b, c^{2}-d b^{4}\right\rangle}=\left\langle a b-c, a c-b^{3} d, c^{2}-b^{4} d\right\rangle=\langle b, c\rangle \cap\left\langle c-a b, a^{2}-b^{2} d\right\rangle$

\footnotetext{
${ }^{1}$ Instead of the relation $c^{2}-b d^{4}$, we might equally well have used $c^{2}-b d^{3}$, in which case $\overline{\mathrm{q}}(\mathrm{c})=1 \frac{1}{2}$. We preferred $\overline{\mathrm{q}}(\mathrm{c})=2$ to emphasize that the advantages of $\overline{g r} \mathrm{R}$ over gr $\mathrm{R}$ are not merely due to the rational grading.
} 
Let $R=\mathbb{F}[a, b, c, d] / I$. Then $R$ has no nilpotents, and neither does $R /\langle b\rangle=$ $\mathbb{F}[\mathrm{a}, \mathrm{b}, \mathrm{c}, \mathrm{d}] /\langle\mathrm{b}, \mathrm{c}\rangle$. But $\mathrm{q}(\mathrm{c})=1, \mathrm{q}\left(\mathrm{c}^{2}\right)=4$, so $\mathrm{c}$ gives a nilpotent element of $\operatorname{gr} R_{1}$.

In fact $\bar{q}(c)=2$, and

$$
\overline{g r} R=\mathbb{F}\left[a_{(0)}, b_{(1)}, c_{(2)}, d_{(0)}\right] /\left\langle a b, a c, c^{2}-b^{4} d\right\rangle
$$

where the parenthesized subscripts indicate the degrees. This, too, can be checked with proposition 4 .

A more standard Gröbner basis calculation tells us

$$
\operatorname{gr} R=\mathbb{F}\left[a_{(0)}, b_{(1)}, c_{(0)}, d_{(0)}\right] /\left\langle c, a^{2} b\right\rangle .
$$

Geometrically, the map gr $\mathrm{R} \rightarrow \overline{\mathrm{gr}} \mathrm{R}$ corresponds (in reverse) to a union of a plane and a surface along a line, mapping to a union of a plane and a double plane along a line, where the map is generically $1: 1$ on the first component and $2: 1$ on the second.

\section{Some normal CONES AND BALANCED nORMAl CONES AS Flat Limits}

Let $R=\mathbb{F}\left[a_{1}, \ldots, a_{n-1}, b\right]$ be a polynomial ring in $n$ variables, and I a radical ideal. Let $Y=\mathbb{A}^{n}=$ Spec $R$, and let $X=\mathbb{A}^{n-1}$ be the $b=0$ hyperplane. Let $V=$ Spec $R / I$, and $W=X \cap V$. We interpret the "basic construction" in this case in terms of a transparent geometric limit, and under the hypotheses of proposition 4 , do the same for the balanced version. We include this description only for illustration, and in this section do not give full proofs (though they are quite straightforward from the theory of Gröbner degenerations).

The basic construction, in this case, goes from

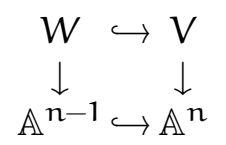

to

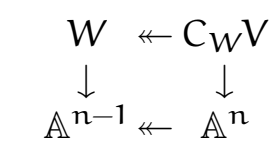

Hence $C_{W} V$ maps into the pullback $W \times \mathbb{A}^{1}$, inducing a Chow class on $W \times \mathbb{A}^{1}$ and thereby on the intersection $W$.

There is a geometric picture of the passage to the normal cone $C_{\mathbb{A}^{n-1}} \mathbb{A}^{n} \cong \mathbb{A}^{n}$. Let the circle $\mathbb{G}_{m}$ act on $\mathbb{A}^{n}$ by

$$
t \cdot\left(a_{1}, \ldots, a_{n-1}, b\right):=\left(a_{1}, \ldots, a_{n-1}, t b\right) .
$$

Then $C_{W} V$ can be computed as the flat $\operatorname{limit} \lim _{t \rightarrow \infty} t \cdot V$, stretching $V$ away from $V \cap \mathbb{A}^{n-1}$.

Two things can happen to any particular component $\mathrm{K} \subseteq \mathrm{V}$ under this limit. If $K \subseteq \mathbb{A}^{n-1}$, then $t \cdot K=K$ for all $t$ including $t=\infty$. The map $C_{W} V \rightarrow W \times \mathbb{A}^{1}$ restricts to a map $\mathrm{K} \rightarrow \mathrm{K} \times \mathbb{A}^{1}$, inducing the zero Chow class. 
It is more interesting when $K \nsubseteq \mathbb{A}^{n-1}$. Then $\lim _{t \rightarrow \infty} t \cdot K=\left(K \cap \mathbb{A}^{n-1}\right) \times \mathbb{A}^{1}$, and the map $C_{W} V \rightarrow W \times \mathbb{A}^{1}$ restricts to an isomorphism $\left(K \cap \mathbb{A}^{n-1}\right) \times \mathbb{A}^{1} \cong(K \cap$ $\left.\mathbb{A}^{n-1}\right) \times \mathbb{A}^{1}$, inducing the fundamental class. The Thom-Gysin isomorphism then takes that to the fundamental class of $K \cap \mathbb{A}^{n-1}$ inside $W$.

In all, the intersection class on $W$ is given by the fundamental classes of the ( $\operatorname{dim} V-1$ )-dimensional components of $W$ (weighted by their lengths), leaving out those components that were components of $\mathrm{V}$.

In the balanced basic construction,

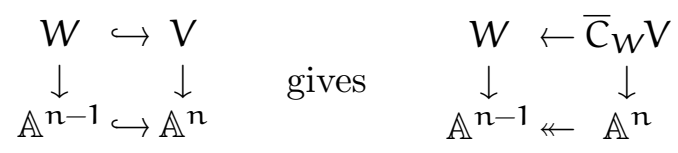

Assume now we are in the case of proposition 4 , where $\bar{q}\left(a_{i}\right)=w_{i}$ for $i=$ $1, \ldots, n-1$, and $\bar{q}$ is induced from the filtration $\bar{q}\left(b^{B} \prod_{i} a_{i}^{n_{i}}\right)=B+\sum_{i} n_{i} w_{i}$. Fix a number $\mathrm{N}>0$ such that each $\mathrm{N} w_{i} \in \mathbb{N}$.

In this case $\bar{C}_{W} V$ can also be computed as a limit. Let $\mathbb{G}_{m}$ act on $\mathbb{A}^{n}$ by

$$
t \cdot\left(a_{1}, \ldots, a_{n-1}, b\right):=\left(t^{N w_{1}} a_{1}, \ldots, t^{N w_{n-1}} a_{n-1}, t^{N} b\right) .
$$

Then it is not hard to show that $\overline{\mathrm{C}}_{W} \mathrm{~V} \cong \lim _{\mathrm{t} \rightarrow \infty} t \cdot \mathrm{V}$.

Example 8. Let $\mathrm{V}$ be the parabola $\left\{\mathrm{b}=\mathrm{a}^{2}\right\}$ in the $\mathrm{ab}$-plane, so $\mathrm{W}$ is $\mathrm{a}$ double point at the origin. Then $t \cdot V$ is the skinny parabola $\left\{b / t=a^{2}\right\}$, whose limit as $t \rightarrow \infty$ is a double line. The map $C_{W} V \rightarrow W \times \mathbb{A}^{1}$ is an isomorphism.

In the balanced construction, $\bar{q}(a)=1 / 2$, and we need $N$ even. So $t \cdot V$ is the parabola $\left\{\mathrm{b} / \mathrm{t}^{\mathrm{N}}=\left(\mathrm{a} / \mathrm{t}^{\mathrm{N} / 2}\right)^{2}\right\}$, which is to say, $\mathrm{t} \cdot \mathrm{V}=\mathrm{V} \cong \overline{\mathrm{C}}_{W} \mathrm{~V}$. The map $\bar{C}_{W} V \rightarrow W \times \mathbb{A}^{1}$ is a double cover of the reduction of $W \times \mathbb{A}^{1}$.

Example 9. Recall the nodal cubic $\mathrm{V}=\operatorname{Spec} \mathrm{R}, \mathrm{R}=\mathbb{F}[\mathrm{a}, \mathrm{b}] /\left\langle\mathrm{b}^{2}-\mathrm{a}^{2}(\mathrm{a}+1)\right\rangle$ from example 5 . The limit picture of the usual normal cone stretches this nodal cubic vertically, resulting in a line at $a=-1$ and a double line at $a=0$.

In this case gr $\mathrm{R}$ was not generated by two variables; we needed to introduce $c=a(a+1)$. Geometrically, $V$ is stretched into the third dimension. In terms of the $\mathbb{R}$-picture, the points in $W=V \cap\{b=0\}$ are left alone, the points elsewhere in $a<0$ are pushed behind the page, and the points in $a>0$ are pulled out of the page. The local picture of an $\times$ through the origin is rotated a bit about the $b$ axis, leaving the ab-plane.

The limit picture of the balanced normal cone stretches not only the vertical dimension, but the new third dimension ( since $\bar{q}(c)=1)$. In the limit, one has a vertical line through the point $(-1,0,0) \in W$, and the 
local picture of an $\times$ has been stretched to a union of two lines lying in the $\mathrm{a}=0$ plane.

It would be interesting to study the relation of balanced normal cones and dynamical intersection theory (which in the context of this section, defines the intersection as the flat limit of $\mathrm{V} \cap\{\mathrm{b}=\mathrm{t}\}$ as $\mathrm{t} \rightarrow 0$ ). Very preliminary investigation suggests that where usual dynamical intersection theory studies how solutions collide as $t \rightarrow 0$, the balanced version keeps track also of how fast they collide.

\section{The Cartier Case: the Ring $\widetilde{\text { gr }}$ And a homotopy interpretation}

We saw in proposition 3 that the multiplication operator $\mathrm{b}$. on $\overline{\operatorname{gr}} \mathrm{R}$ is always $1: 1$ above degree 0 , so that

$$
\overline{\mathrm{gr}} \mathrm{R} \longrightarrow \mathrm{R} / \sqrt{\mathrm{I}} \oplus \overline{\mathrm{gr}} \mathrm{R} / \mathrm{ann}(\mathrm{b})
$$

is an injection. This proposition also told us that on $\overline{g r} \mathrm{R} / \mathrm{ann}(\mathrm{b})$, multiplying by $\mathrm{b}$ is $1: 1$ and in high degrees, onto. That suggests that we fill in the holes in small degrees, which we do now.

The map $\overline{g r} \mathrm{R}$ to the fraction ring $\overline{\mathrm{gr}} \mathrm{R}\left[\mathrm{b}^{-1}\right]$ has kernel ann(b). Define

$$
\widetilde{\mathrm{gr}} \mathrm{R}:=\text { the integral closure of } \overline{\mathrm{gr}} \mathrm{R} / \mathrm{ann}(\mathrm{b}) \text { in } \overline{\mathrm{gr}} \mathrm{R}\left[\mathrm{b}^{-1}\right] \text {. }
$$

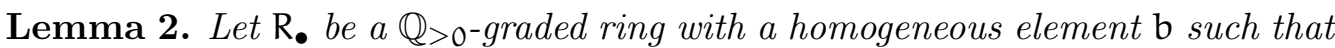
$\mathrm{b} \cdot: \mathrm{R}_{\mathrm{n}} \rightarrow \mathrm{R}_{\mathrm{n}+1}$ is $1: 1$ for all $\mathrm{n}$ and onto for large $\mathrm{n}$. Let $\mathrm{r} \in \mathrm{R}_{\mathrm{k}}$ be homogeneous. Then $\mathrm{r} / \mathrm{b}\lfloor\mathrm{k}\rfloor$ is integral over $\mathrm{R}$, and even over $\mathrm{R}_{0}[\mathrm{~b}]$.

Proof. Pick $d>0$ such that $k d \in \mathbb{N}$. Then $r^{d} \in R_{k d}$, and to show $r$ is integral it is enough to show $r^{d}$ is integral. In this way we can reduce to the case $k \in \mathbb{N}$, which we assume hereafter.

Fix $N \in \mathbb{N}$ such that $b \cdot: R_{n} \rightarrow R_{n+1}$ is onto for all $n \geq N$. Then $R_{N}$ is a finite module over $R_{0}$ (proof: take a homogeneous generating set for the ideal $\oplus_{n \geq N} R_{n}$; the elements in $R_{N}$ generate $R_{N}$ as an $R_{0}$-module). Since we can use $b$. to identify all these $R_{n}$ for $n \geq N, n \in \mathbb{N}$, we will denote this module by $R_{\mathbb{N} \gg 0}$. By multiplying by $b^{N}$, any homogeneous element $s \in R$ has an image $s^{\prime}$ in $R_{\mathbb{N} \gg 0}$.

Now consider the sequence $1^{\prime}, r^{\prime},\left(r^{2}\right)^{\prime}, \ldots$ in $R_{\mathbb{N} \gg 0}$. They generate an $R_{0^{-}}$ submodule of $R_{\mathbb{N} \gg 0}$, but only finitely many are needed to generate. Hence for some $m>0$, we can write $\left(r^{m}\right)^{\prime}=\sum_{i<m} c_{i}\left(r^{i}\right)^{\prime}$ with each $c_{i} \in R_{0}$. Lifting back to $R$, this becomes $r^{m}=\sum_{i<m} c_{i} b^{k(m-i)} r^{i}$. So $r$ satisfies a monic polynomial with $R_{0}[b]$-coefficients.

Theorem 3. Let $\mathrm{R}$ be a ring and $\mathrm{b}$ an element, inducing $\overline{\mathrm{q}}, \overline{\mathrm{gr}} \mathrm{R}, \widetilde{\mathrm{gr}} \mathrm{R}$ as above. 
Then the natural map

$$
\overline{\mathrm{gr}} \mathrm{R} \rightarrow \mathrm{R} / \sqrt{\mathrm{I}} \oplus \widetilde{\mathrm{gr}} \mathrm{R}
$$

is a graded inclusion, and an isomorphism in high degrees. The multiplication map

$$
\mathrm{b} \cdot \widetilde{\mathrm{gr}} \mathrm{R}_{\mathrm{n}} \rightarrow \widetilde{\mathrm{gr}} \mathrm{R}_{\mathrm{n}+1}
$$

is an isomorphism for all rational $\mathrm{n} \geq 0$.

(A common reflex is to conclude from this that $\widetilde{\mathrm{gr}} \mathrm{R} \cong\left(\widetilde{\mathrm{gr}} \mathrm{R}_{0}\right)[\mathrm{b}]$. But $\widetilde{\mathrm{gr}} \mathrm{R}$ is rationally graded, not integrally, so this result is merely specifying a periodicity in the grading.)

If $\mathrm{R}^{\bullet}$ is a graded ring with $\mathrm{R}^{0}=\mathbb{F}$ an algebraically closed field, and $\mathrm{b} \in \mathrm{R}^{\bullet}$ is homogeneous for this grading, then $\widetilde{\text { gr }} \mathrm{R}$ splits naturally as a finite direct sum of doubly graded rings $\left\{A_{i}\right\}_{i=1 \ldots m}$ with each $\left(A_{i}\right)^{0}=\left(A_{i}\right)_{0}^{0} \cong \mathbb{F}$.

Proof. The map given is the composite

$$
\overline{\mathrm{gr}} \mathrm{R} \rightarrow \mathrm{R} / \sqrt{\mathrm{I}} \oplus \overline{\mathrm{gr}} \mathrm{R} / \mathrm{ann}(\mathrm{b}) \rightarrow \mathrm{R} / \sqrt{\mathrm{I}} \oplus \widetilde{\mathrm{gr}} \mathrm{R}
$$

of two graded inclusions (taking $\mathrm{R} / \sqrt{\mathrm{I}}$ to be degree 0 ), and hence is one also.

Using proposition 3, choose $N>0$ such that $b \cdot: \overline{g r} R_{n} \rightarrow \overline{g r} R_{n+1}$ is an isomorphism for all $n \geq N$. Then if $c / b^{k} \in \widetilde{\text { gr }} R_{n}$ for $n \geq N$, we know $c \in$ $(\overline{g r} R / \operatorname{ann}(b))_{n+k}$, which we can identify with $\overline{g r} R_{n+k}$ since $n+k \geq N+k \geq N>0$. Then by the assumption on $N, c=b^{k} d$ for some $d$, so $c / b^{k}=d \in \overline{g r} R_{n}$. This shows that the inclusion $\overline{\text { gr }} R_{n} \rightarrow \widetilde{\text { gr }} R_{n}$ is onto for $n \geq N$.

To see that $b$. is an isomorphism for all rational $n \geq 0$, we apply lemma 2 to gr R.

Since $\widetilde{\text { gr }} \mathrm{R}$ stands between $\overline{\mathrm{gr}} \mathrm{R} / \operatorname{ann}(\mathrm{b})$ and its full normalization, it is finite over $\overline{\text { gr }} \mathrm{R} / \operatorname{ann}(\mathrm{b})$. In particular $\widetilde{\operatorname{gr}} \mathrm{R}^{0}$ is a finite-dimensional $\mathbb{F}$-algebra. Since $\widetilde{\text { gr }} \mathrm{R}^{0}$ has no nilpotents and $\mathbb{F}$ is algebraically closed, we find $\widetilde{\mathrm{gr}} \mathrm{R}^{0} \cong \oplus_{i} \mathbb{F}$. In more detail, $\widetilde{\text { gr }} R^{0}$ has a unique $\mathbb{F}$-basis $\left(\pi_{1}, \ldots, \pi_{m}\right)$ up to reordering, with $\pi_{i}^{2}=\pi_{i}, \pi_{i} \pi_{j}=0$ for $i \neq j$.

Again since $\widetilde{\text { gr }} \mathrm{R}^{0}$ is finite-dimensional without nilpotents, all of $\widetilde{\mathrm{gr}} \mathrm{R}^{0}$ must be in $\widetilde{\operatorname{gr}} R_{0}^{0}$.

Let $A_{i}=\pi_{i} \widetilde{g r} R$ as an algebra with unit element $\pi_{i}$. Then $\widetilde{g r} R=\oplus A_{i}$ as claimed.

It was to obtain a theorem like this that first led the author to the study of balanced normal cones, to study the Hilbert function of $R^{\bullet}$ in terms of $R / \sqrt{I}$ and gr R. In a future publication $[\mathrm{Kn}]$ we will use theorem 3 inductively to study standard bases of homogeneous coordinate rings. 
For the rest of this section, we make the assumptions of the latter part of the theorem, namely that $R^{\bullet}$ is a graded ring with $R^{0}=\mathbb{F}$ an algebraically closed field, and $b \in R^{\bullet}$ is a homogeneous element. Let $Y=\operatorname{Proj} R^{\bullet}$ and $X=\operatorname{Proj} R /\langle b\rangle$ the divisor $b=0$. Let $\bar{C}_{X}^{c} Y=\operatorname{Proj} \overline{g r} R^{\bullet}$, where the ${ }^{c}$ is there as reminder that this is a completion of the usual cone. ${ }^{2}$ Write $\widetilde{C}_{X}^{c} Y$ for Proj $\widetilde{g r} R$. Then by the last part of the theorem above, $\widetilde{C}_{X}^{c} Y$ is a disjoint union of weighted cones $\{$ Proj $A\}$.

We can now interpret some of these ring maps geometrically:

$$
\begin{aligned}
& \begin{array}{llrl}
\overline{\operatorname{gr}} \mathrm{R} \rightarrow \overline{\operatorname{gr}} \mathrm{R}\left[\mathrm{b}^{-1}\right] & \Longleftrightarrow & \overline{\mathrm{C}}_{X}^{\mathrm{c}} Y \backslash X \hookrightarrow \overline{\mathrm{C}}_{X}^{\mathrm{c}} Y \\
\overline{\operatorname{gr}} \mathrm{R} \hookrightarrow \mathrm{R} / \sqrt{\mathrm{I}} \oplus \overline{\operatorname{gr}} \mathrm{R} / \operatorname{ann}(\mathrm{b}) & \Longleftrightarrow & X_{\text {red }} \cup \overline{\overline{\mathrm{C}}_{X}^{\mathrm{c}} Y \backslash X} \rightarrow \overline{\mathrm{C}}_{X}^{\mathrm{c}} Y
\end{array} \\
& \overline{\operatorname{gr}} \mathrm{R} \hookrightarrow \mathrm{R} / \sqrt{\mathrm{I}} \oplus \bigoplus_{\mathrm{A}} A \quad \Longleftrightarrow \quad X_{\text {red }} \cup \cup_{A} \operatorname{Proj} A \rightarrow \overline{\mathrm{C}}_{X}^{\mathrm{C}} Y
\end{aligned}
$$

\subsection{Examples.}

Example 10. Recall $\mathrm{R}=\mathbb{F}[\mathrm{a}, \mathrm{b}] /\left\langle\mathrm{a}^{2}-\mathrm{ab}\right\rangle$ from example 7 , with $\overline{\mathrm{q}}(\mathrm{a})=$ $\overline{\mathrm{q}}(\mathrm{b})=1$.

Here $f=a b^{-1}$ is integral, since $f(f-1)=b^{-2} a(a-b)=0$, and $\widetilde{\mathrm{gr}} \mathrm{R}=\mathbb{F}[\mathrm{b}, \mathrm{f}] /\langle\mathrm{f}(\mathrm{f}-1)\rangle \cong \mathbb{F}[\mathrm{b}] \oplus \mathbb{F}\left[\mathrm{b}^{\prime}\right]$.

Geometrically, the divisor $\mathrm{b}=0$ is a double point at the intersection of the two lines Spec R. The normal cone Spec gr R is the trivial line bundle over the double point. The balanced normal cone $\operatorname{Spec} \operatorname{gr} R \cong \operatorname{Spec} R$ is just the two intersecting lines. Whereas Spec $\widetilde{g r} R$ pulls apart the two lines; it is the full normalization.

Example 11. Let $\mathrm{R}=\mathbb{F}[\mathrm{b}, \mathrm{c}, \mathrm{d}] /\left\langle\mathrm{c}\left(\mathrm{c}^{2}-\mathrm{bd}\right)\right\rangle$, so $\mathrm{X}=$ Proj $\mathrm{R}$ is the union of a line and a conic in the plane. One of the two points of intersection lies on the line $b=0$, which is tangent to the conic there. Using proposition 4 , we find $\overline{\operatorname{gr}} \mathrm{R} \cong \mathrm{R}$, with $\mathrm{b} \in \overline{\operatorname{gr}} \mathrm{R}_{1}, \mathrm{c} \in \overline{\operatorname{gr}} \mathrm{R}_{1 / 2}, \mathrm{~d} \in \overline{\operatorname{gr}} \mathrm{R}_{0}$.

Then $f=c^{2} b^{-1} \in \overline{g r} R\left[b^{-1}\right]$ is integral, because $f(f-d)=c b^{-2} c\left(c^{2}-\right.$ bd) $=0$. In fact

$$
\widetilde{\operatorname{gr} R}=\mathbb{F}[b, c, d, f] /\left\langle c(f-d), b f-c^{2}, f(f-d)\right\rangle,
$$

so Proj $\widetilde{g r} R /\langle b\rangle$ is the disjoint union of the point Proj $\widetilde{\mathrm{gr}} R /\langle b, c\rangle$ and the (doubly fat) point Proj $\widetilde{g r} R /\left\langle b, f-d, c^{2}\right\rangle$. Whereas Proj $\overline{g r} R /\langle b\rangle$ is only one (triply fat) point.

Note that Proj $\widetilde{\text { gr }} \mathrm{R}$ is not the full normalization of $\mathrm{X}$, which would pull the two components apart at both ends.

Example 12. Recall the ring $\mathrm{R}=\mathbb{F}[\mathrm{b}, \mathrm{c}, \mathrm{d}, \mathrm{e}] /\langle\mathrm{d}(\mathrm{b}-\mathrm{d}), e(\mathrm{~b}-\mathrm{d}), \mathrm{dc}, e \mathrm{c}\rangle$ from subsection 2.2.2, the union of the $d=e=0$ plane and $b-d=c=0$

\footnotetext{
${ }^{2}$ Note that this Proj is defined using the original grading $R^{\bullet}$ on $R$ (which descends to one on $\overline{g r} R$ since $b$ is homogeneous), not the new grading $\overline{g r} R_{\bullet}$. We won't need this latter space Projgr $R_{\bullet}$, whose non-balanced analogue Projgr $R_{\bullet}$ is the exceptional divisor in the blowup of Spec $R$ along Spec $R /\langle b\rangle$, hence isomorphic to $\operatorname{Spec} R /\langle b\rangle$.
} 
plane. We found that the associated graded gr $\mathrm{R}$ turns out to be isomorphic to $R$, with $\bar{q}(b)=\bar{q}(d)=1, \bar{q}(c)=\bar{q}(e)=0$.

The first few graded pieces of gr $R_{\bullet}$ are

$$
\begin{aligned}
& \overline{\operatorname{gr}} R_{0}=\mathbb{F}[c, e] /\langle e c\rangle \\
& \overline{\operatorname{gr}} R_{1}=b\left(\overline{\operatorname{gr}} R_{0}\right) \oplus \mathbb{F} d \\
& \overline{\operatorname{gr}} R_{2}=b^{2}\left(\overline{\operatorname{gr}} R_{0}\right) \oplus \mathbb{F} b d \\
& \overline{\operatorname{gr}} R_{3}=b^{3}\left(\overline{g r} R_{0}\right) \oplus \mathbb{F} b^{2} d
\end{aligned}
$$

so $\bar{b} \cdot: R_{0} \rightarrow R_{1}$ is $1: 1$, and is an isomorphism in all higher degrees. This suggests we look at the element $f=d b^{-1}$. It is indeed integral, satisfying $f(f-1)=0$.

We know that $\widetilde{\text { gr }} \mathrm{R}$ should be the b-cone over $\widetilde{\text { gr }} \mathrm{R}_{0}$, so should have no relations involving $b$; each will end up replaced by relations in degree 0 . In fact

$$
\widetilde{g r} R=\mathbb{F}[b, c, f, e] /\langle f(f-1), e(f-1), f c, e c\rangle,
$$

geometrically the cone over the disjoint union of the two lines $c=f-1=0$, $e=f=0$.

Example 13. From subsection 2.2.3, recall the ring

$$
\overline{\operatorname{gr}} \mathrm{R}=\mathbb{F}\left[\mathrm{a}_{(0)}, \mathrm{b}_{(1)}, \mathrm{c}_{(2)}, \mathrm{d}_{(0)}\right] /\left\langle\mathrm{ab}, \mathrm{ac}, \mathrm{c}^{2}-\mathrm{b}^{4} \mathrm{~d}\right\rangle
$$

where the parenthesized subscripts indicate the degrees. The first few graded pieces are

$$
\begin{aligned}
& \overline{\operatorname{gr}} R_{0}=\mathbb{F}[a, d] \\
& \overline{\operatorname{gr}} R_{1}=b\left(\overline{g r} R_{0}\right) \\
& \overline{\operatorname{gr}} R_{2}=b^{2}\left(\overline{g r} R_{0}\right) \oplus c\left(\overline{g r} R_{0}\right) \\
& \overline{\operatorname{gr}} R_{3}=b^{3}\left(\overline{g r} R_{0}\right) \oplus b c\left(\overline{g r} R_{0}\right) \\
& \overline{g r} R_{4}=b^{4}\left(\overline{g r} R_{0}\right) \oplus b^{2} c\left(\overline{g r} R_{0}\right)
\end{aligned}
$$

In this example $b$ is a zero divisor, and $\bar{b} \cdot: \overline{g r} R_{0} \rightarrow \overline{g r} R_{1}$ is not $1: 1$. All later maps are $1: 1$, but only become onto at and after $\overline{\mathrm{gr}} \mathrm{R}_{2} \rightarrow \overline{\mathrm{gr}} \mathrm{R}_{3}$.

The relation $c^{2}-b^{4} d=0$ says that $e=c b^{-2}$ is integral, as $e^{2}=d$. In fact

$$
\widetilde{\mathrm{gr} R}=\mathbb{F}[\mathrm{a}, \mathrm{b}, e, \mathrm{~d}] /\left\langle\mathrm{a}, \mathrm{e}^{2}-\mathrm{d}\right\rangle
$$

where we've lost the component that lived in $b=0$.

Note that this is an example where the inclusion $\overline{\operatorname{gr}} R \rightarrow \widetilde{\mathrm{gr}} R$ is not an isomorphism in all positive degrees $n-$ only $n \geq 2$. (That's because $\overline{\mathrm{q}}(\mathrm{c})=2$; this phenomenon is unrelated to $\mathrm{b}$ being a zero divisor.) 
Example 14. This is an irreducible example with $\widetilde{\mathrm{gr}} \mathrm{R} \neq \overline{\mathrm{gr}} \mathrm{R}$.

Consider $R=\mathbb{F}[b, c, d, f] /\left\langle b^{2} f+b c d+c^{3}\right\rangle$, the homogeneous coordinate ring of a cubic surface. It has a $\mathbb{P}^{1}$ of singularities, along $\mathrm{b}=\mathrm{c}=0$. It is easy to see that $c^{2 n+1} \in\left\langle b^{n}\right\rangle$, and the lower bounds $\bar{q}(c) \geq 1 / 2$, $\bar{q}(d), \bar{q}(f) \geq 0$ suggest the degeneration $\mathbb{F}\left[b_{(1)}, c_{(1 / 2)}, d_{(0)}, f_{(0)}\right] /\left\langle b c d+c^{3}\right\rangle$. Applying proposition 4 , we see that we have correctly computed $\overline{\mathbf{q}}$, and gr R.

The first few graded pieces of $\overline{g r} \mathrm{R}$ are

$$
\begin{aligned}
\overline{\operatorname{gr}} R_{0} & =\mathbb{F}[d, f] \\
\overline{\operatorname{gr}} R_{\frac{1}{2}} & =\mathbb{F}[d, f] c \\
\overline{g r} R_{1} & =\mathbb{F}[d, f] b \oplus \mathbb{F}[d, f] c^{2} \\
\overline{\operatorname{gr}} R_{1 \frac{1}{2}} & =\mathbb{F}[d, f] b c \\
\overline{\operatorname{gr}} R_{2} & =\mathbb{F}[d, f] b^{2} \oplus \mathbb{F}[d, f] b c^{2}
\end{aligned}
$$

Multiplication by $b$ should give a "1-fold periodicity" on $\widetilde{\text { gr }} \mathrm{R}$, suggesting we let $y=c^{2} b^{-1}$ fill in the hole seen in degree 0 . Then $y^{2}+d y=$ $b^{-2} c\left(c^{3}+b c d\right)=0$, so $y$ is indeed integral over R. In fact

$\widetilde{\mathrm{gr}} \mathrm{R}=\mathbb{F}[\mathrm{b}, \mathrm{c}, \mathrm{d}, \mathrm{f}, \mathrm{y}] /\left\langle\mathrm{y}^{2}+\mathrm{d} y, \mathrm{by}-\mathrm{c}^{2}\right\rangle=(\mathbb{F}[\mathrm{d}, \mathrm{f}, \mathrm{y}] /\langle\mathrm{y}(\mathrm{y}+\mathrm{d})\rangle)[\mathrm{b}, \mathrm{c}] /\left\langle\mathrm{b} y-\mathrm{c}^{2}\right\rangle$.

Consider the map $\overline{\mathrm{gr}} \mathrm{R} \hookrightarrow \widetilde{\mathrm{gr}} \mathrm{R}$ after $\mathrm{b}$ is killed:

$$
\mathbb{F}[c, d, f] /\left\langle c^{3}\right\rangle \rightarrow \mathbb{F}[c, d, f, y] /\left\langle y^{2}+d y, c^{2}\right\rangle
$$

Taking Proj, this is a map from a bouquet of two $\mathbb{P}^{1} \mathrm{~s}$ (one with multiplicity 2) onto a single $\mathbb{P}^{1}$ (with multiplicity 3 ), $1: 1$ at the north pole intersection but otherwise $2: 1$.

In case it helps give intuition into $\widetilde{\text { gr }}$, we observe that the integral closure can be taken before or after $\overline{\mathrm{gr}}$.

Proposition 7. Let $\mathrm{R}$ be a ring and $\mathrm{b} \in \mathrm{R}$. Let $\widetilde{\mathrm{R}}$ denote the integral closure of $\mathrm{R} / \mathrm{ann}(\mathrm{b})$ in $\mathrm{R}\left[\mathrm{b}^{-1}\right]$. Then $\widetilde{\operatorname{gr}} \mathrm{R} \cong \overline{\mathrm{gr}} \widetilde{\mathrm{R}}$.

Proof. First, define a filtration on $\mathrm{R}\left[\mathrm{b}^{-1}\right]$ again called $\overline{\mathrm{q}}$ by $\overline{\mathrm{q}}\left(\mathrm{p} / \mathrm{b}^{\mathrm{k}}\right)=\overline{\mathrm{q}}(\mathrm{pb})-$ $(k+1)$. By Rees' formula for $\bar{q}$, this formula is well-defined. (If $b$ is not a zero divisor, the more obvious formula $\overline{\mathbf{q}}(p)-k$ works as well.) Plainly it restricts to $\bar{q}$ on $R / \operatorname{ann}(b)$, justifying the reuse of the name. With it, we can define $\overline{g r}\left(R\left[b^{-1}\right]\right)$, easily seen to be isomorphic to $(\overline{\mathrm{gr}} \mathrm{R})\left[\mathrm{b}^{-1}\right]$. Now the $\overline{\mathrm{gr}}$ of

$\mathrm{R} / \operatorname{ann}(\mathrm{b}) \hookrightarrow \widetilde{\mathrm{R}} \hookrightarrow \mathrm{R}\left[\mathrm{b}^{-1}\right] \quad$ gives $\quad \overline{\mathrm{gr}}(\mathrm{R} / \operatorname{ann}(\mathrm{b})) \hookrightarrow \overline{\mathrm{gr}} \widetilde{\mathrm{R}} \hookrightarrow \overline{\mathrm{gr}}\left(\mathrm{R}\left[\mathrm{b}^{-1}\right]\right)=(\overline{\mathrm{gr}} \mathrm{R})\left[\mathrm{b}^{-1}\right]$.

Our goal is to show that $\overline{g r} \widetilde{R}$ is the integral closure of $\overline{g r}(R / \operatorname{ann}(b))$ in $\overline{g r}\left(R\left[b^{-1}\right]\right)$. 
We first show $\overline{g r} \widetilde{R}$ is integral over gr $R$. Let $r \in R$ lie over $\bar{r} \in \overline{g r} R$. Then if $r / b^{k}$ satisfies a monic polynomial $p \in R[x]$, its image $\bar{r} / b^{k}$ satisfies $\bar{p} \in \overline{g r} R[x]$. Hence we have maps

$$
\overline{\mathrm{gr}}(\mathrm{R} / \mathrm{ann}(\mathrm{b})) \hookrightarrow \overline{\mathrm{gr}} \widetilde{\mathrm{R}} \hookrightarrow \widetilde{\mathrm{gr}} \mathrm{R} .
$$

Since the composite is an isomorphism in large degrees, so is the second inclusion.

Now it is enough to know that the map b : $\overline{\mathrm{gr}} \widetilde{\mathrm{R}}_{\mathrm{n}} \rightarrow \overline{\mathrm{gr}} \widetilde{\mathrm{R}}_{\mathrm{n}+1}$ is onto for all $n \geq 0$. Let $\bar{p} \in \overline{g r} \widetilde{R}_{n+1}$ be the image of $p \in \widetilde{R}_{n+1}$, so $\bar{q}(p)=n+1 \geq 1$. Then by Rees' formula and the valuative criterion for integrality, $p / b$ is integral over $R / \operatorname{ann}(b)$, so $p / b \in \widetilde{R}_{n}$, giving a preimage of $\bar{p}$ in $\overline{\text { gr }} \widetilde{R}_{n}$.

4.2. A homotopical analogy. Let $X$ be a topological space, and D a closed subset. Then we can think of $X$ as built from $D$ with $\overline{X \backslash D}$ attached. A standard homotopical operation at this point is to study $(X, X \backslash D)$ by collapsing $X \backslash D$ to a point, or at least something contractible.

That is slightly too brutal for us. First thicken $D$ to an open neighborhood $D_{+}$such that $D_{+}$retracts to $D$ and $X \backslash D$ retracts to $X \backslash D_{+}$. Then separate $X \backslash D$ into connected components $W$, with each $W_{-}:=W \backslash D_{+}$a closed retract of $W$. Now let $X^{\prime}$ be $X$ with each $W_{-}$collapsed to its own point. In good cases, up to homotopy this means we replace $\bar{W}$ with the cone on $\bar{W} \cap \mathrm{D}$.

Example 15. Let $\mathrm{X}$ be a circle and $\mathrm{D}$ a point on it. Then $\mathrm{X} \backslash \mathrm{D}$ is connected, so there is only one connected component $W$, and $W$ is already contractible. But in the (trivial) passage from $X$ to $X^{\prime}$ we don't replace $W$ by the cone on the point $\bar{W} \backslash W=X \backslash(X \backslash D)=D$; that would flatten the circle $X$ to an interval. Rather, we replace $W$ with the cone on $\overline{W_{-}} \cap \overline{D_{+}}$, which is two points.

In bad cases like this example, we are still replacing $W$ with a cone - it's just not the cone on $\bar{W} \cap \mathrm{D}$, but instead a sort of link of that inside $\bar{W}$. In the above example, the link was two points.

In the algebraic geometry, the passage from $X \rightarrow X^{\prime}$ parallels the flat degeneration $R \rightarrow \overline{g r} R$. The decomposition of the open set $X^{\prime} \backslash D$ into connected components $\cup W$ corresponds to the decomposition of the fraction ring $\overline{g r} R\left[b^{-1}\right]$ as a direct sum $\bigoplus_{A} A$. "Being a cone" is replaced by having a periodic grading.

The most subtle point in the above topological picture is the fact that we don't replace each $\bar{W}$ with a cone on $\bar{W} \cap \mathrm{D}$, but on something that maps to $\bar{W} \cap \mathrm{D}$. In the algebraic geometry, this reflects the fact that the inclusion $\overline{g r} R / \operatorname{ann}(b) \hookrightarrow \widetilde{\text { gr } R}$ may not be an isomorphism. In this way, perhaps one should think of the map gr $\mathrm{R} / \mathrm{ann}(\mathrm{b}) \hookrightarrow \widetilde{\mathrm{gr}} \mathrm{R}$ as sort of an attaching map when building a complex. In example 14 above, the attaching map is the one from the bouquet of two $\mathbb{P}^{1} \mathrm{~s}$ to a single $\mathbb{P}^{1}$. 


\section{From local to global}

One can define the normal cone for any pair $\mathrm{W} \subseteq \mathrm{V}$ of schemes, not necessarily affine. The cleanest way to do this is to take global Spec [Ht, ex. 5.17] of the sheaf of $\mathcal{O}_{V}$-algebras gr $\mathcal{O}_{V}:=\mathcal{O}_{V} / \mathcal{I} \oplus \mathcal{I} / \mathcal{I}^{2} \oplus \ldots$ where $\mathcal{I}$ is the ideal sheaf of the subscheme $W$.

To define the balanced normal cone for any pair $\mathrm{W} \subseteq \mathrm{V}$ (where $\mathrm{V}$ is now reduced), we need an analogue of this sheaf of algebras. To each open set $\mathrm{U}$, we have associated filtrations $\mathrm{qu}, \overline{\mathrm{q}}_{\mathrm{u}}$ on $\Gamma\left(\mathrm{U}_{;} \mathcal{O}_{\mathrm{v}}\right)$. Let

$$
\Gamma\left(\mathrm{U} ; \mathcal{O}_{\mathrm{V}}\right)_{\mathrm{n}}:=\left\{\mathrm{r} \in \Gamma\left(\mathrm{U} ; \mathcal{O}_{\mathrm{V}}\right): \overline{\mathrm{q}}(\mathrm{r}) \geq \mathrm{n}\right\}, \quad \mathrm{n} \in \mathbb{Q} .
$$

This is obviously a presheaf of $\mathcal{O}_{\mathrm{V}}$-ideals. For each $\mathrm{U}$, there is a number $\mathrm{N}_{\mathrm{U}}$ such that $\mathrm{N}_{\mathrm{U}} \overline{\mathrm{q}}_{\mathrm{u}}$ is integral, i.e. $\Gamma\left(\mathrm{U} ; \mathcal{O}_{\mathrm{V}}\right)_{\mathfrak{n}}$ only depends on $\left\lfloor\mathrm{N}_{\mathrm{U}} \mathfrak{n}\right\rfloor$.

Proposition 8. Let $\mathrm{W} \subseteq \mathrm{V}$ where $\mathrm{V}$ is a reduced scheme. Then each presheaf $\mathrm{U} \mapsto \Gamma\left(\mathrm{U} ; \mathcal{O}_{\mathrm{V}}\right)_{n}$ is a sheaf. Consequently we can define the sheaf of graded algebras

$$
\overline{\operatorname{gr}} \mathcal{O}_{\mathbf{V}} \quad \text { defined by } \mathrm{U} \mapsto \bigoplus_{\mathrm{n} \in \mathbb{Q}}\left(\Gamma\left(\mathrm{U} ; \mathcal{O}_{\mathbf{V}}\right)_{\mathfrak{n}} / \sum_{n_{+}>\mathrm{n}} \Gamma\left(\mathrm{U} ; \mathcal{O}_{\mathbf{V}}\right)_{n_{+}}\right)
$$

whose Spec we call the balanced normal cone $\overline{\mathrm{C}}_{W} \mathrm{~V}$.

Proof. Since these presheaves are defined as sub-presheaves of $\mathcal{O}_{\mathrm{V}}$, they automatically satisfy the uniqueness half of the gluing axiom for sheaves, so we focus on existence. Let $\mathrm{r}_{1} \in \Gamma\left(\mathrm{U}_{1} ; \mathcal{O}_{\mathrm{V}}\right)_{n}, \mathrm{r}_{2} \in \Gamma\left(\mathrm{U}_{2} ; \mathcal{O}_{\mathrm{V}}\right)_{n}$ agree on $\mathrm{U}_{1} \cap \mathrm{U}_{2}$, and let $r \in \Gamma\left(U_{1} \cup U_{2} ; \mathcal{O}_{V}\right)$ be their common extension. We must show $\bar{q}_{u_{1} \cup U_{2}}(r) \geq n$.

Let $n_{-}=n-\epsilon<n$. Since $r_{i} \in \Gamma\left(U_{1} ; \mathcal{O}_{V}\right)_{n}$ for $i=1,2$, there exist $M_{i}>0$ such that $q_{u_{i}}\left(r_{i}^{M_{i}}\right)>M_{i} n_{-}$. So $q_{u_{i}}\left(r_{i}^{M_{1} M_{2}}\right)>M_{1} M_{2} n_{-}$, indeed $q_{u_{i}}\left(r_{i}^{m M_{1} M_{2}}\right)>$ $m M_{1} M_{2} n_{-}$for all integral $m>0$. Since the ideal sheaf $\mathcal{I}^{\left\lfloor m M_{1} M_{2} n_{-}\right\rfloor}$is indeed a sheaf, we learn that $q_{u_{1}} \cup u_{2}\left(r^{m M_{1} M_{2}}\right)>\left\lfloor m M_{1} M_{2} n_{-}\right\rfloor$for all integral $m>0$. Hence $\bar{q}_{\mathrm{u}_{1} \cup \mathrm{u}_{2}}(\mathrm{r})>\mathrm{n}_{-}$. Since $\epsilon$ was arbitrary, we find $\overline{\mathrm{q}}_{\mathrm{u}_{1} \cup \mathrm{u}_{2}}(\mathrm{r}) \geq \mathrm{n}$.

The construction of the (usual) normal cone as a Spec has a parallel construction of the blowup as a Proj. However, we cannot directly apply this Proj construction to the obvious sheaf $\mathrm{U} \mapsto \bigoplus_{n \in \mathbb{Q}} \Gamma\left(\mathrm{U} ; \mathcal{O}_{V}\right)_{n}$ in order to define a "balanced blowup", because the Proj construction really requires the algebras to be $\mathbb{N}$-graded.

We could work with the sheaf $U \mapsto \bigoplus_{n \in \frac{1}{N} \mathbb{N}} \Gamma\left(U ; \mathcal{O}_{V}\right)_{n}$ for some $N>0$, but this only interfaces well with the balanced normal cone construction if each stalk of $\overline{g r} \mathcal{O}_{V}$ is $\frac{1}{N} \mathbb{N}$-graded. We now show how to find such an $\mathrm{N}$ in the case $\mathrm{V}$ is of finite type. 
Lemma 3. Let $\mathrm{I} \leq \mathrm{R}$ be an ideal, and $\mathrm{q}_{\mathrm{R}}, \overline{\mathrm{q}}_{\mathrm{R}}$ the associated filtrations. Let $\mathrm{c} \in \mathrm{R}$, and let $\mathrm{S}$ be the fraction ring $\mathrm{R}\left[\mathrm{c}^{-1}\right]$, with filtrations $\mathrm{q}_{\mathrm{S}}, \overline{\mathrm{q}}_{\mathrm{S}}$ associated to the ideal $\mathrm{I}\left[\mathrm{c}^{-1}\right]$. Then for each $\mathrm{r} \in \mathrm{R}$, either $\mathrm{q}_{\mathrm{S}}(\mathrm{r})=\infty$ or $\mathrm{q}_{\mathrm{S}}(\mathrm{r})=\mathrm{q}_{\mathrm{R}}\left(\mathrm{rc}^{\mathrm{m}}\right)$ for some $\mathrm{m}$.

In particular, if $\mathrm{N}_{\mathrm{R}}, \mathrm{N}_{\mathrm{S}}$ denote the LCMs of the denominators of $\overline{\mathrm{q}}_{\mathrm{R}}, \overline{\mathrm{q}}_{\mathrm{S}}$, then $\overline{\mathrm{q}}_{S} \mid \overline{\mathrm{q}}_{\mathrm{R}}$.

Proof. For any $n$,

$$
\begin{aligned}
\overline{\mathrm{q}}_{S}(\mathrm{r})>\mathrm{n} & \Longleftrightarrow \forall M \gg 0, \mathrm{r}^{M} \in\left(\mathrm{I}\left[\mathrm{c}^{-1}\right]\right)^{\lfloor n M\rfloor} \\
& \Longleftrightarrow \exists \mathrm{m}, \forall M \gg 0,\left(\mathrm{rc}^{\mathrm{m}}\right)^{M} \in \mathrm{I}^{\lfloor n M\rfloor} \\
& \Longleftrightarrow \exists \mathrm{m}, \overline{\mathrm{q}}_{\mathrm{R}}\left(\mathrm{rc}^{\mathrm{m}}\right)>\mathrm{n} \\
& \Longleftrightarrow \lim _{\mathrm{m} \rightarrow \infty} \overline{\mathrm{q}}_{\mathrm{R}}\left(\mathrm{rc}^{\mathrm{m}}\right)>\mathrm{n} \quad \text { since this is increasing in } \mathrm{m}
\end{aligned}
$$

Hence $\bar{q}_{S}(r)=\lim _{m \rightarrow \infty} \bar{q}_{R}\left(r^{m}\right)$. The weakly increasing sequence $\bar{q}_{R}\left(r^{m}\right)$ takes a discrete set of values, so if it doesn't go to infinity then it achieves its limit at some finite $\mathrm{m}$.

(We leave the reader to convince herself that in the case that I is principal, this lemma also follows easily from Rees' formula.)

Proposition 9. Let $\mathrm{W} \subseteq \mathrm{V}$ be a closed subscheme and $\mathrm{V}$ be of finite type. Then there is a number $\mathrm{N}>0$ such that each stalk of $\overline{\text { gr }} \mathcal{O}_{\vee}$ is $\frac{1}{\mathrm{~N}} \mathbb{N}$-graded.

Proof. To find the $\mathrm{N}$, we cover $\mathrm{V}$ by finitely many affine charts $\mathrm{U}_{\alpha}$, and let $\mathrm{N}$ be the least common multiple of the $\mathrm{N}_{\mathrm{U}_{\alpha}}$ for those charts. Now we need to show that $\mathrm{N}$ has the desired property.

For each $v \in V$, choose $\alpha$ such that the affine chart $\mathrm{U}_{\alpha}$ contains $v$. Now we show that for any affine $\mathrm{U} \subseteq \mathrm{U}_{\alpha}, \mathrm{N}_{\mathrm{U}} \mid \mathrm{N}_{\mathrm{U}_{\alpha}}$, or equivalently that $\mathrm{N}_{\mathrm{U}_{\alpha}} \bar{q}_{\mathrm{U}}$ is integer-valued (where $\bar{q}_{\mathrm{u}}$ is the homogenized filtration on $\Gamma\left(\mathrm{U} ; \mathcal{O}_{V}\right)$ ). Proof: let $\mathrm{c} \in \Gamma\left(\mathrm{U}_{\alpha} ; \mathcal{O}_{\mathrm{V}}\right)$ be a function vanishing on $\mathrm{U}_{\alpha} \backslash \mathrm{U}$ (necessarily a divisor, since $\mathrm{U}$ is affine). Then apply lemma 3.

Now note that affine open sets contained in $\mathrm{U}_{\alpha}$ give a basis for the topology at $v$, so they are enough to compute the stalk.

In this case we christen $\operatorname{Proj}\left(\mathrm{U} \mapsto \bigoplus_{n \in \frac{1}{N} \mathbb{N}} \Gamma\left(\mathrm{U} ; \mathcal{O}_{V}\right)_{n}\right)$ the balanced blowup of $\mathrm{V}$ along $\mathrm{W}$. It would be interesting to understand what universal property characterizes balanced blowups.

\section{Proof of theOREM 1}

Since we have to deal seriously with Chow classes in this section, we list some simple properties we will need of them: 
(1) If $\phi: W \rightarrow V$ is proper, there is an induced map $\phi_{*}: A_{\bullet}(W) \rightarrow A_{\bullet}(V)$ of their Chow groups, and these maps are functorial.

(2) The inclusion $W_{\text {red }} \hookrightarrow W$ of the reduction of $W$ induces a map $A .\left(W_{\text {red }}\right) \rightarrow$ A. $(W)$ which is an isomorphism.

(3) Any sheaf $\mathcal{F}$ on $V$ determines a class in $A_{\operatorname{dim} V}(V)$, namely the sum $\sum_{C \subseteq V}\left(\operatorname{dim} \mathcal{F}_{C}\right)[C]$ where $C$ ranges over the top-dimensional ${ }^{3}$ components of $\mathrm{V}$. In particular, the structure sheaf $\mathcal{O}_{V}$ defines the fundamental class $[\mathrm{V}] \in A_{\operatorname{dim} V}(\mathrm{~V})$. This assignment descends to a map $\mathrm{K}_{\bullet}(\mathrm{V}) \rightarrow$ $A_{\operatorname{dim}} \mathrm{V}(\mathrm{V})$ from K-homology.

(This is only the simplest approximation to a better-known result: the K-homology possesses a natural filtration whose associated graded space is rationally isomorphic to $A(V)$. The above approximation is all we will need.)

Because of property 2 , we can work on any scheme whose reduction is $\left(\mathrm{C}_{W} \mathrm{~V}\right)_{\text {red }}$. This will make it easier to find a venue in which the two induced classes in Khomology are equivalent. From there, we will use property 3 to deduce that the two induced Chow classes are equivalent.

The following K-homology lemma also appears in [AK], where it is applied to a more general situation than the one considered in this paper.

Proposition 10. Let $\mathrm{t}$ be a non-zero-divisor in $\mathrm{Q}$, and let $\overline{\mathrm{Q}} \geq \mathrm{Q}$ stand between $\mathrm{Q}$ and its integral closure in $\mathrm{Q}\left[\mathrm{t}^{-1}\right]$. Assume that $\overline{\mathrm{Q}}$ is finite over $\mathrm{Q}$ (for example, if $\mathrm{Q}$ is reduced and finitely generated over a field).

Then $\exists \mathrm{N}>0$ such that the modules $\mathrm{Q} / \mathrm{tQ}$ and $\overline{\mathrm{Q}} / \mathrm{t} \overline{\mathrm{Q}}$ define the same element of $\mathrm{K}$-homology of the ring $\mathrm{Q} /\left\langle\mathrm{t}^{\mathrm{N}}\right\rangle$.

Proof. Consider the short exact sequences of Q-modules

$$
\mathrm{O} \rightarrow \mathrm{Q} / \mathrm{tQ} \rightarrow \overline{\mathrm{Q}} / \mathrm{tQ} \rightarrow \overline{\mathrm{Q}} / \mathrm{Q} \rightarrow \mathrm{0}, \quad \mathrm{O} \rightarrow \mathrm{t} \overline{\mathrm{Q}} / \mathrm{tQ} \rightarrow \overline{\mathrm{Q}} / \mathrm{tQ} \rightarrow \overline{\mathrm{Q}} / \mathrm{t} \overline{\mathrm{Q}} \rightarrow 0
$$

Since $t$ is not a zero divisor, the natural map $\bar{Q} / Q \rightarrow t \bar{Q} / t Q$ is an isomorphism. So we get the equation in K-homology

$$
[\mathrm{Q} / \mathrm{tQ}]=[\overline{\mathrm{Q}} / \mathrm{tQ}]-[\overline{\mathrm{Q}} / \mathrm{Q}]=[\overline{\mathrm{Q}} / \mathrm{tQ}]-[\mathrm{t} \overline{\mathrm{Q}} / \mathrm{tQ}]=[\overline{\mathrm{Q}} / \mathrm{t} \overline{\mathrm{Q}}] .
$$

By the assumptions on $\bar{Q}$, there exists an $N$ such that $t^{N-1} \bar{Q} \subset Q$. Therefore $\mathrm{t}^{\mathrm{N}}$ annihilates all of these modules, so they are modules over the ring $\mathrm{Q} /\left\langle\mathrm{t}^{\mathrm{N}}\right\rangle$, and the derivation of this K-homology equation holds there.

\footnotetext{
${ }^{3}$ Some authors define the fundamental Chow class as a sum over all components, but this definition has some problems. Here is one. Given a connected flat family of projective schemes, one can push forward the fundamental Chow classes of the fibers to give Chow classes on projective space. Our definition gives a constant result, whereas the sum over all components may not.
} 
Before continuing the proof, we try to give some topological feeling for the application of property 2. Given four spaces $A, B \subseteq X \subseteq Y$, knowing $A$ and $B$ are homologous in $X$ implies they are homologous in $Y$. We can go the reverse direction (knowing $A$ and $B$ are homologous in $Y$ implies they are homologous in $X$ ) if $X$ is a homotopy retract of $Y$. But $Y$ may be a more convenient place to calculate. Property 2 says that, when computing Chow classes, $W_{\text {red }}$ behaves like a homotopy retract of $W$.

Corollary 1. In the setup of proposition 10, the class in $A_{\text {top }}(\operatorname{Spec} Q /\langle t\rangle)$ associated to the module $\overline{\mathrm{Q}} / \mathrm{t} \overline{\mathrm{Q}}$ is the fundamental class of Spec $\mathrm{Q} /\langle\mathrm{t}\rangle$.

Proof. The result follows from the above proposition, property 3, and two applications of property 2, comparing $\mathrm{Q} /\left\langle\mathrm{t}^{\mathrm{N}}\right\rangle$ to $\mathrm{Q} / \sqrt{\left\langle\mathrm{t}^{\mathrm{N}}\right\rangle}=\mathrm{Q} / \sqrt{\langle\mathrm{t}\rangle}$ to $\mathrm{Q} /\langle\mathrm{t}\rangle$.

Example 16. We give an example to show how things can fail if $\mathrm{Q}$ is not reduced. Consider the ring $\mathbb{F}\left[x, t, t^{-1}\right] /\left\langle x^{2}\right\rangle$, graded by power of $t$, and let $\mathrm{Q}$ be the graded subring generated by $t$ and $x t^{-1}$. So the degree $n$ part is 0 for $n<-1, \mathbb{F} \times t^{-1}$ for $n=-1$, and $\mathbb{F} t^{n} \oplus \mathbb{F} \times t^{n}$ for $n \geq 0$.

The integral closure $\bar{Q}$ of $\mathrm{Q}$ inside $\mathrm{Q}\left[\mathrm{t}^{-1}\right]=\mathbb{F}\left[x, t, \mathrm{t}^{-1}\right] /\left\langle\mathrm{x}^{2}\right\rangle$ agrees with $\mathrm{Q}$ in degrees $\mathrm{n} \geq-1$, but in degrees $\mathrm{n}<-1$ it includes also $\mathbb{F} \times \mathrm{t}^{\mathrm{n}}$, as each $x t^{n}$ satisfies the monic polynomial $z^{2}=0$. Since $\bar{Q}$ uses all negative degrees and $\mathrm{Q}$ is supported in degree $\mathrm{n} \geq-1, \overline{\mathrm{Q}}$ is not a finitely generated module over $\mathrm{Q}$. Moreover, the conclusion of corollary 1 is false; $\overline{\mathrm{Q}} / \mathrm{t} \overline{\mathrm{Q}} \cong \mathbb{F}$ gives the class of a single point, whereas $\operatorname{Spec} Q /\langle t\rangle \cong \mathbb{F}\left[x t^{-1}\right] /\left\langle\left(x t^{-1}\right)^{2}\right\rangle$ is a double point.

We will obtain the integrality required in the above proposition from the I-adic case of Theorem 4.16, part iii, of [Re]:

Theorem 4. $[\mathrm{Re}]$ Let $\mathrm{R}$ be a Noetherian ring, $\mathrm{I}$ an ideal, and $\mathrm{q}, \overline{\mathrm{q}}$ as in the introduction. Define $\mathrm{q}^{*}(\mathrm{r})$ as the maximum $\mathrm{m}$ such that $\mathrm{r}$ satisfies a monic polynomial

$$
x^{n}+a_{1} x^{n-1}+\ldots+a_{n}=0
$$

with each $\mathbf{q}\left(a_{i}\right) \geq \mathbf{i m}$. Then $\mathbf{q}^{*}=\lfloor\overline{\mathbf{q}}\rfloor$.

Proof of theorem 1. We first reduce to the affine case as follows. While Chow classes on a scheme are in principle global objects, those in top degree are local in that they are characterized by their restriction to the generic point of each (top-dimensional) component. And each of those generic points is hit by $\mathrm{C}_{\mathrm{X}} \mathrm{Y}$ for some affine opens $\mathrm{X} \subseteq \mathrm{W}, \mathrm{Y} \subseteq \mathrm{V}$.

By hypothesis, we can work in affine charts where the coordinate ring $\mathrm{R}$ of $\mathrm{Y}$ is finitely generated over a field. Let $\mathrm{I}$ be the ideal defining $\mathrm{X}$, and define $\mathrm{q}, \overline{\mathrm{q}}$, gr $\mathrm{R}, \overline{\mathrm{gr}} \mathrm{R}$ as usual. 
Let $\mathrm{N}$ be the LCM of the denominators in $\overline{\mathrm{q}}$. Then define two subalgebras of $R\left[t, t^{-1}\right]$, where $t$ is a new indeterminate;

$$
\mathrm{Q}=\bigoplus_{n \in \mathbb{Z}} \mathrm{t}^{-n}\{r: q(r) \geq n / N\} \quad \bar{Q}=\bigoplus_{n \in \mathbb{Z}} t^{-n}\{r: \bar{q}(r) \geq n / N\}
$$

(If $N=1$, then the first is exactly the Rees algebra of the pair R, I.) It is easy to see that $\mathrm{Q} /\langle\mathrm{t}\rangle=\operatorname{gr} \mathrm{R}, \overline{\mathrm{Q}} /\langle\mathrm{t}\rangle=\overline{\mathrm{gr}} \mathrm{R}$, once one rescales the grading by $-\mathrm{N}$. To connect to the statement given of theorem 1 , we observe that the corresponding schemes are $\mathrm{C}_{\mathrm{X}} \mathrm{Y}, \overline{\mathrm{C}}_{\mathrm{X}} \mathrm{Y}$, which are patches on $\mathrm{C}_{W} \mathrm{~V}, \overline{\mathrm{C}}_{W} \mathrm{~V}$.

In either $\mathrm{Q}$ or $\overline{\mathrm{Q}}$, the positive degree summands are just $\mathrm{R}$, so $\mathrm{Q}\left[\mathrm{t}^{-1}\right]=$ $\overline{\mathrm{Q}}\left[\mathrm{t}^{-1}\right]=\mathrm{R}\left[\mathrm{t}, \mathrm{t}^{-1}\right]$. In particular, $\overline{\mathrm{Q}} \leq \mathrm{Q}\left[\mathrm{t}^{-1}\right]$. Since $\overline{\mathrm{q}} \geq \mathrm{q}$, we also have a containment $\mathrm{Q} \leq \overline{\mathrm{Q}}$. We are almost in the situation of proposition 10; it remains only to show that $\bar{Q}$ is integral over $\mathrm{Q}$. (Finiteness follows from the fact that $\mathrm{Q}$ too is reduced and finitely generated over a field, hence has a finite normalization.)

Let $s=t^{-k} r \in \bar{Q}_{k}$, so $\bar{q}(r) \geq k / N$. The element $r$ is integral over $Q$ iff its $N$ th power is, so by replacing $s$ by its $N$ th power we can reduce to the case that $k$ is a multiple of $N$. Hence $q^{*}(r) \geq k / N$, as defined in theorem 4 above. So $r$ satisfies a monic polynomial

$$
r^{n}+a_{1} r^{n-1}+a_{2} r^{n-2}+\ldots+a_{n}=0 \quad \text { with each } q\left(a_{i}\right) \geq i k / N .
$$

Dividing by $t^{\text {nk }}$, we get

$$
s^{n}+\left(a_{1} / t^{k}\right) s^{n-1}+\left(a_{2} / t^{2 k}\right) s^{n-2}+\ldots+a_{n} / t^{n k}=0
$$

Since $q\left(a_{i}\right) \geq i k / N$, each $a_{i} / t^{i k}$ is in $Q$, so $s$ is integral over $Q$ as was to be shown.

We are now able to apply corollary 1, which tells us that the class in $A_{\text {top }}$ (Spec $\operatorname{gr} \mathrm{R}$ ) associated to the module $\overline{\mathrm{gr}} \mathrm{R}$ is the fundamental class of Spec gr R.

The proof is done; we pause to unwind it. By property $2, A_{\text {top }}\left(\left(C_{X} Y\right)_{\text {red }}\right)=$ $A_{\text {top }}\left(C_{X} Y\right)$. Using that identification and the last paragraph above, we showed that the class in $A_{\text {top }}\left(\left(C_{X} Y\right)_{\text {red }}\right)$ associated to the thickening $C_{X} Y$ matches the class in $A_{\text {top }}\left(\left(C_{X} Y\right)_{\text {red }}\right)$ associated to the branched cover $\bar{C}_{X} Y$. As explained in the first paragraph, we can use local calculations like this to establish the desired result in $A_{\text {top }}\left(\left(C_{W} V\right)_{\text {red }}\right)$.

Example 17. Since theorem 1 uses proposition 10, it can fail if $Y$ is not reduced. Let $R=\mathbb{F}[x] /\left\langle x^{2}\right\rangle, I=\langle x\rangle$. Then $\mathrm{q}(\mathrm{x})=1$, and $\overline{\mathrm{q}}(\mathrm{x})=\infty$. So

$$
\mathrm{Q}=\bigoplus_{\mathrm{n} \in \mathbb{Z}} \mathrm{t}^{-\mathrm{n}}\{\mathrm{r}: \mathrm{q}(\mathrm{r}) \geq \mathrm{n}\} \quad \overline{\mathrm{Q}}=\bigoplus_{\mathrm{n} \in \mathbb{Z}} \mathrm{t}^{-\mathrm{n}}\{\mathrm{r}: \overline{\mathrm{q}}(\mathrm{r}) \geq \mathrm{n}\}
$$

are the $\mathrm{Q}, \overline{\mathrm{Q}}$ of example 16 . 
After observing this Rees algebra normalization interpretation of theorem 4, we found the same interpretation in Theorem 10.6.6 of the forthcoming book $[\mathrm{HuSw}]$.

\section{REFERENCES}

[Al] V. Alexeev, Complete moduli in the presence of semiabelian group action, Ann. of Math. (2) 155 (2002), no. 3, 611-708. math.AG/9905103

[AK] V. Alexeev, A. Knutson, Complete moduli spaces of branchvarieties. math. AG/0602626

[Ei] D. Eisenbud, Commutative algebra, with a view toward algebraic geometry, Graduate Texts in Mathematics, 150. Springer-Verlag, New York, 1995.

[FM] W. Fulton, R. MacPherson, Intersecting cycles on an algebraic variety, Real and complex singularities (Proc. Ninth Nordic Summer School/NAVF Sympos. Math., Oslo, 1976), pp. 179-197, Sijthoff and Noordhoff, Alphen aan den Rijn, 1977.

[Hr] J. Harris, Galois groups of enumerative problems, Duke Math. J. 46 (1979), no. 4, 685-724.

[Ht] R. Hartshorne, Algebraic geometry, Springer-Verlag, New York, 1977, Graduate Texts in Mathematics, No. 52.

[HuSw] C. Huneke, I. Swanson, Integral Closure of Ideals, Rings, and Modules, in preparation. Available at http://www.reed.edu/ iswanson/book/

[Kn] A. Knutson, Standard bases for homogeneous coordinate rings, in preparation.

[LJT] M. Lejeune-Jalabert, B. Teissier, Clôture intégrale des idéaux et équisingularité, unpublished.

[M2] D. Grayson, M. Stillman, Macaulay 2, a software system for research in algebraic geometry, available at http://www.math.uiuc.edu/Macaulay2/

[Na] M. Nagata, Note on a paper of Samuel concerning asymptotic properties of ideals, Mem. Coll. Sci. Univ. Kyoto. Ser. A. Math. 30 (1957), 165-175.

[Re] D. Rees, Lectures on the asymptotic theory of ideals, London Mathematical Society Lecture Note Series, 113, Cambridge University Press, Cambridge, 1988.

[Sa] P. Samuel, Some asymptotic properties of powers of ideals, Ann. of Math. (2) 56 (1952), $11-21$.

Allen Knutson

9500 Gilman Drive

Dept 0112

La Jolla, CA 92093-0112

USA

E-mail: allenk@math.ucsd.edu 